\title{
Phytochemistry and Diverse Pharmacology of Genus Mimosa: A Review
}

\author{
Komal Rizwan ${ }^{1, *}$, Ismat Majeed ${ }^{2}$, Muhammad Bilal ${ }^{3}$ (D) Tahir Rasheed $^{4}\left(\mathbb{D}\right.$, Ahmad Shakeel ${ }^{5, *(D)}$ \\ and Shahid Iqbal ${ }^{6}$
}

check for updates

Citation: Rizwan, K.; Majeed, I.; Bilal, M.; Rasheed, T.; Shakeel, A.; Iqbal, S. Phytochemistry and Diverse Pharmacology of Genus Mimosa: A Review. Biomolecules 2022, 12, 83. https://doi.org/10.3390/ biom12010083

Academic Editors: Jen-Tsung Chen and Jose M. Prieto

Received: 19 November 2021

Accepted: 4 January 2022

Published: 5 January 2022

Publisher's Note: MDPI stays neutral with regard to jurisdictional claims in published maps and institutional affiliations.

Copyright: (C) 2022 by the authors. Licensee MDPI, Basel, Switzerland. This article is an open access article distributed under the terms and conditions of the Creative Commons Attribution (CC BY) license (https:// creativecommons.org/licenses/by/ $4.0 /)$.
1 Department of Chemistry, University of Sahiwal, Sahiwal 57000, Pakistan

2 Department of Chemistry, Government College Women University, Faisalabad 38000, Pakistan; ismatmajeed123@gmail.com

3 School of Life Science and Food Engineering, Huaiyin Institute of Technology, Huaian 223003, China; bilaluaf@hyit.edu.cn

4 Interdisciplinary Research Center for Advanced Materials, King Fahd University of Petroleum and Minerals (KFUPM), Dhahran 31261, Saudi Arabia; masil@sjtu.edu.cn

5 Department of Hydraulic Engineering, Faculty of Civil Engineering and Geosciences, Delft University of Technology, Stevinweg 1, 2628 CN Delft, The Netherlands

6 Department of Chemistry, School of Natural Sciences (SNS), National University of Science and Technology (NUST), H-12, Islamabad 46000, Pakistan; shahidiqbal.chem@sns.nust.edu.pk

* Correspondence: komal.rizwan45@yahoo.com (K.R.); a.shakeel@tudelft.nl (A.S.)

\begin{abstract}
The genus Mimosa belongs to the Fabaceae family and comprises almost 400 species of herbs, shrubs and ornamental trees. The genus Mimosa is found all over the tropics and subtropics of Asia, Africa, South America, North America and Australia. Traditionally, this genus has been popular for the treatment of jaundice, diarrhea, fever, toothache, wound healing, asthma, leprosy, vaginal and urinary complaints, skin diseases, piles, gastrointestinal disorders, small pox, hepatitis, tumor, $\mathrm{HIV}$, ulcers and ringworm. The review covered literature available from 1959 to 2020 collected from books, scientific journals and electronic searches, such as Science Direct, Web of Science and Google scholar. Various keywords, such as Mimosa, secondary metabolites, medicines, phytochemicals and pharmacological values, were used for the data search. The Mimosa species are acknowledged to be an essential source of secondary metabolites with a wide-ranging biological functions, and up until now, 145 compounds have been isolated from this genus. Pharmacological studies showed that isolated compounds possess significant potential, such as antiprotozoal, antimicrobial, antiviral, antioxidant, and antiproliferative as well as cytotoxic activities. Alkaloids, chalcones, flavonoids, indoles, terpenes, terpenoids, saponins, steroids, amino acids, glycosides, flavanols, phenols, lignoids, polysaccharides, lignins, salts and fatty esters have been isolated from this genus. This review focused on the medicinal aspects of the Mimosa species and may provide a comprehensive understanding of the prospective of this genus as a foundation of medicine, supplement and nourishment. The plants of this genus could be a potential source of medicines in the near future.
\end{abstract}

Keywords: genus; Mimosa; plant; phytochemicals; biological molecules; pharmacological activities

\section{Introduction}

The Mimosa genus is a member of the legumes family Fabaceae (subfamily: mimosoideae) and consists of about 400 species of shrubs and herbs [1]. Almost 20-25 species of this genus are well known to the world including Mimosa tenuiflora (Wild.) pior, Mimosa pudica L., Mimosa pigra L., Mimosa caesalipiniifolia Benth., Mimosa hamata Willd., Mimosa diplotricha Sauvalle, Mimosaa xanthocentra Mart., Mimosa artemisiana Heringer \& Paula, Mimosa invisa Mart. ex Colla, Mimosa scabrella Benth., Mimosa somnians Humb. \& Bonpl. ex Willd., Mimosa bimucronata (DC.) Kuntze, Mimosa verrucosa Benth., Mimosa arenosa (Willd.) Poir., Mimosa humilis Willd., Mimosa rubicaulis Lam., Mimosa linguis and M. albida Willd., and Mimosa ophthalmocentra Mart. ex Benth. (http:/ / mpns.kew.org/MPNS.kew.org accessed 
on 12 December 2018; www.theplantlist.org accessed on 12 December 2018). These species are found in Brazil [2], Africa (Mauritius, Nigeria and Reunion), numerous Pacific Islands, North America, Papua New Guinea [3], Australia [4], Mexico, Venezuela [5], Thailand and the Philippines [6]. However, they are mainly found in Asia, such as Pakistan [7,8], Japan, Indonesia, India, Bangladesh, Malaysia [9], India [10], China, Sri Lanka, Taiwan and Cambodia, while certain species are commonly distributed from Cuba to Texas, northern Central America, Paraguay, Uruguay and Argentina, [11]. The species of Mimosa grow in diverse habitats, such as the savannas, lowland tropical rainforests, dry forests and thorn scrubs of tropical and subtropical areas, midelevations of subtropical deserts, forests, wetlands and grasslands [12]. The leaves of the Mimosa species may be binate or pinnate. Some species such as M. pudica and M. pigra show response to touch by folding their leaves. Flowers may be white, globular pink and in the form of clusters. The fruits are brittle, and a wall of the fruit is compacted in the middle of the seeds. The root bark contains large amounts of starch and calcium oxalate crystals $[13,14]$.

The plants of this genus especially are employed for ornamental purposes and also serve as a sleeping shelter for animals [15]. Various species of the Mimosa genus are socially and economically important, such as M. scabrella (timber production), M. caesalpiniifolia (reforestation) and $M$. tenuiflora (source of firewood). These plants are also used for flooring and furniture [16,17]. The leaves of the plants are used for chicken diet [18], as colorants in the textile industry and also play a role as additives in the food industry $[19,20]$. The trash of the poultry industry enhances the growth of the Mimosa tree [21,22]. The Mimosa genus has significant economic status in the cosmetic industry [23]. Phytochemical studies of this genus revealed the presence of flavonoids, steroids, saponins, alkaloids, coumarins, tannins [24,25] and terpenoids [26]. The genus Mimosa showed several pharmacological activities, such as antiseptic, antimicrobial [27,28], antidiabetic [29,30], antihistamic [31], antioxidants [32-37], anticonvulsant [38], antigout [39], antispasmolytic [40], anti-inflammatory [41-43], antinociceptive [44,45], antiulcer [43], antifertility [46], antimalarial [47], antiparasitic [48], wound healing [49], anticancer [50,51], antidepressant [52], antidiarrheal [53,54], hypolipidemic [55,56], hepatoprotective [57], antivenom [43], antiproliferative [58], antiviral [59] and aphrodisiac [60]. Previously our group efficiently documented the indigenous flora of Pakistan and also a few exotic species [61-75]. This review covered the several review articles as well in the discussion regarding this genus [76-80]. Recently, our group reported the ethotraditional uses and pharmacological potential of the crude extracts of different species of the genus Mimosa [81]. In this review, we comprehensively reported the secondary metabolites isolated from the genus Mimosa and their pharmacological potential along with future perspectives.

\section{Materials and Methods}

A detailed bibliographic study that included papers published from 1959 to 2020 was conducted. A number of databanks (Web of Science, Science Direct, SciFinder, Francis \& Taylor, Scopus, SciELO, Google Scholar, Springer, PubMed, Wiley, Google and The Plant-Database) were surveyed for assembling statistics, data and figures for this genus. A number of related books, complete text documents and summaries were checked. The genus name, synonyms and scientific names of the genus Mimosa species were castoff as the keywords. The scientific name of all the plants of genus Mimosa and its substitutes were corroborated by consuming a standard databank (http:/ / mpns.kew.org/MPNS.kew.org accessed on 12 December 2018; www.theplantlist.org accessed on 12 December 2018).

\section{Chemical Profiling of Genus Mimosa}

\subsection{Qualitative and Quantitative Analysis of Phytochemicals in Genus Mimosa}

Haddad et al. determined condensed tannins of $M$. tenuiflora whose active ingredients were procyanidin and prodelphinidins [82]. Oliveira et al. determined the total phenols (TP; 99.29 and 65.37), total tannins (TT; 65.57 and 54.93) and condensed tannins (CT; 34.56 and 30.98) from the leaves and stems of M. tenuiflora, respectively [83]. Racadio reported the 
existence of phytochemicals in the EtOH extract of $M$. pudica leaves. Phytochemicals, such as alkaloids, flavonoids, saponins and triterpenes, were present, while sterols and tannins were found to be absent [84]. Ahuchaogu et al. screened the phytochemicals of the EtOH extract of the whole M. pudica plant. The ethanol extract consisted of alkaloids (9.05\%), flavonoids $(8.32 \%)$, steroids $(2.49 \%)$, saponins $(8.15 \%)$, phenols $(1.02 \%)$, tannins $(0.083 \%)$, cyanogenic glycosides $(0.122 \%)$ and anthocyanins $(1.913 \%)$ [85]. Durgadevi and Karthika reported the phytochemicals of the aq. extract of $M$. pudica leaves. A qualitative analysis indicated the presence of flavonoids, alkaloids, proteins, steroids, tannins, saponins and terpenoids, while phlobatannins, phenols and reducing sugars were found to be absent. The quantitative phytochemical investigation of saponins, flavonoids, tannins and terpenoids were $0.48 \mathrm{mg} / \mathrm{mL}, 0.99 \mathrm{mg} / \mathrm{mL}, 0.80 \mathrm{mg} / \mathrm{mL}, 0.39 \mathrm{mg} / \mathrm{mL}$ and $0.39 \mathrm{mg} / \mathrm{mL}$ of the aq. extract recorded, respectively [24]. Sheeba et al. screened the phytochemicals of the $\mathrm{MeOH}$ extracts of $M$. pudica leaves. The plant showed the presence of flavonoids, phenols and tannins, while alkaloids, glycosides, terpenoids, amino acids and carbohydrates were found to be absent [86]. Tunna et al. reported the presence of phytochemicals in M. pudica aerial parts. Alkaloids, saponins, flavonoids, terpenoids and coumarins were found to be present, while carotenoids and anthraquinone were found to be absent [29]. Parmar et al. measured the phytochemicals of the EtOH extract of M. pudica roots. Extracts showed the presence of tannins, alkaloids, terpenoids, flavonoids, sterols, phenolic compounds and proteins [34]. Mahadevan et al. measured the phytochemicals of aq. extract of the whole M. pudica plant. A Phytochemical analysis exhibited the existence of alkaloids, flavonoids and tannins [87]. Ramesh et al. investigated phytochemicals of various extracts (EtOH, $\mathrm{MeOH}, \mathrm{PE}$ and ACE) of $M$. pudica leaves and roots. The results showed the presences of flavonoids, alkaloids, terpenoids, carbohydrates, saponins, amino acids, phenols, tannins, proteins and steroids, while glycosides, fats, oils, resins, reducing sugars, phytosterols and phlobatannins were found to be absent [88]. Chinnathambi and Sathasivam measured the phytochemicals of the $\mathrm{ACE}, \mathrm{EtOH}$ and aq. extracts of $M$. pudica leaves. The investigation proved the existence of tannins, terpenoids, phlobatannins, steroids, saponins and glycoside, while flavonoids were absent [89]. Mathew et al. measured the phytochemicals of the $\mathrm{MeOH}$ extracts of the $M$. pudica plant. The results showed the existence of compounds, such as flavonoids alkaloids, saponins, terpenoids, phenols, glycosides, tannins and coumarins [26]. Nagarajan et al. determined the phytochemicals of aq. extract of $M$. pudica leaves and stems by using the Harborne methods $[90,91]$. The phytochemicals, such as saponins, alkaloids, flavonoids, tannins and phenols were present.

Nagarajan et al. determined the quantitative assessment of the mineral contents of the aq. extracts of M. pudica leaves and stems by using wet digestion extraction methods. The mineral contents, such as magnesium, phosphorus, calcium, nitrogen and potassium, were observed [91]. Lee et al. reported the presence of neoxanthin $\left(9.86 \mathrm{ggg}^{-1} \mathrm{FW}\right)$, viola xanthin $\left(6.57 \mu \mathrm{gg}^{-1} \mathrm{FW}\right)$, lutein $\left(7.75 \mu \mathrm{gg}^{-1} \mathrm{FW}\right)$, lycopene $\left(0.62 \mu \mathrm{gg}^{-1} \mathrm{FW}\right)$, carotene $(\alpha=0.19$, $\beta=0.25$ vit. $\left.\mathrm{E}^{-1} \mathrm{~g}\right)$, tocopherol $\left(\alpha=0.25\right.$ vit. $\left.\mathrm{E}^{-1} \mathrm{~g}\right)$, total Carotenoids $\left(25.24 \mu \mathrm{gg}^{-1} \mathrm{FW}\right)$ and total vitamins $\left(0.25 \mathrm{\mu gg}^{-1} \mathrm{FW}\right)$ in leaves of M. pudica [92]. Ittiyavirah and Pullochal determined the phytochemicals of the EtOH extract of the whole $M$. pudica plant. The preliminary phytochemical analysis of $M$. pudica revealed the existence of alkaloids, flavonoids, tannins, phenolics and steroids [93]. Ao et al. and Olusayo et al. screened the preliminary phytochemicals of the EtOH extract of $M$. pigra roots. The tannins, phlobatannins, flavonoids, triterpenes and saponins were found to be present, while alkaloids, anthraquinones and phenolics were found to be absent [94,95]. Rosado-Vallado et al. screened the phytochemicals of the $\mathrm{MeOH}$ and aq. extracts of $M$. pigra leaves. The result showed the existence of flavonoids, quinones, saponins, sterols and tannins [96]. Saxena et al. measured the phytochemicals of the $\mathrm{EtOH}$ and $\mathrm{MeOH}$ extracts of the whole $M$. hamata plant. The preliminary phytochemical analysis indicated the existence of flavonoids, alkaloids, phytosterols, glycosides, tannins, phenolic compounds, saponins and carbohydrates, while proteins and amino acids, fixed oils and fats were found to be absent [97]. Manosroi et al. reported the presence of various phytochemicals, such as flavones, glycosides, saponins alkaloids and 
tannins, in the aq. extract of $M$. invisa leaves, while anthraquinones and xanthones were found to be absent [98]. Jiménez et al. determined the total phenolic contents of the aq. extract of the whole M. albida plant by the Folin-Ciocalteu method. The plant showed a high phenolic content (323 mg GAE/g) [99]. Seraglio et al. measured the phytochemicals of $M$. scabrella bentham honeydew honeys. Coumarins, flavonoids, lignin-derived aldehydes and phenolic acids were found to be present [100]. Nandipati et al. reported on the flavonoids, tannins, triterpenes and carbohydrates in the $\mathrm{MeOH}$ extract of the M. rubicaulis stem [101]. Table 1 presents various phytochemicals present in different species of the genus Mimosa. Phytochemicals are widely known for their medicinal activities. Primary metabolites, such as proteins, lipids and amino acids, are responsible for biochemical reactions in plants, while secondary metabolites, such as saponins, flavonoids, alkaloids, tannins, phenols and glycosides, protect plants against damage and also play a role in the improvement of flavor, color and fragrance. [102]. Phytochemicals in plants are very present in leaves, roots and stems, and their percentage varies because of environmental conditions, plant genus, etc. The Mimosa genus is rich in these primary and secondary metabolites, so the plants of this genus are well known for their pharmacological potential.

Table 1. Phytochemical screening of different species of genus Mimosa.

\begin{tabular}{|c|c|c|c|}
\hline Plant & Plant Part/Extract & Phytochemicals & References \\
\hline \multirow{2}{*}{ M. tenuiflora } & Whole plant & condensed tannins, procyanidin, prodelphinidins & [82] \\
\hline & leaves, stem & total phenols, total tannins and condensed tannins & [83] \\
\hline \multirow{14}{*}{ M. pudica } & Leaves/EtOH extract & alkaloids, flavonois, saponins and triterpenes & [84] \\
\hline & Whole plant/EtOH extract & alkaloids, flavonoids, steroids, saponins, phenols, tannins, cyanogenic & [85] \\
\hline & Leaves/aq. extract & flavonoids, alkaloids, proteins, steroids, tannins, saponins and terpenoids & [24] \\
\hline & Leaves/MeOH extract & alkaloids, glycosides, terpenoids and amino acids & [86] \\
\hline & Aerial parts & alkaloids, saponins, flavonoids, terpenoids and coumarins & [29] \\
\hline & Roots/EtOH extract & $\begin{array}{l}\text { proteins, sterols, tannins, terpenoids, alkaloids, flavonoids and } \\
\text { phenolic compounds }\end{array}$ & [34] \\
\hline & Whole plant/aq. extract & alkaloids, flavonoids and tannins & [87] \\
\hline & $\begin{array}{l}\text { Leaves and roots/EtOH, } \mathrm{MeOH}, \mathrm{PE} \\
\text { and ACE extracts }\end{array}$ & $\begin{array}{l}\text { flavonoids, alkaloids, terpenoids, carbohydrates, saponins, amino acids, phenols, } \\
\text { tannins, proteins and steroids }\end{array}$ & {$[88]$} \\
\hline & Leaves/ACE, EtOH and aq. extracts & tannin, terpenoids, phlobatannins, steroids, saponin and glycoside & [89] \\
\hline & Whole plant/MeOH extract & $\begin{array}{c}\text { flavonoids alkaloids, saponins, terpenoids, phenols, glycosides, tannins, } \\
\text { and coumarins }\end{array}$ & [26] \\
\hline & Leaves and stem/aq. extract & saponins, alkaloids, flavonoids, tannins and phenols & {$[90,91]$} \\
\hline & Leaves and stem/aq. extract & magnesium, phosphorus, calcium, nitrogen and potassium & [91] \\
\hline & Leaves & $\begin{array}{c}\text { neoxanthin, viola xanthin, lutein, lycopene, carotenes, tocopherol, total } \\
\text { carotenoids and total vitamins }\end{array}$ & [92] \\
\hline & Whole plant/EtOH extrac & alkaloids, flavonoids, tannins, phenolics and steroids & [93] \\
\hline M. pigra & $\begin{array}{l}\text { Roots } / \text { EtOH extract } \\
\text { Leaves/MeOH and aq. extracts }\end{array}$ & $\begin{array}{l}\text { tannins, phlobatannins, flavonoids, triterpenes and saponins } \\
\text { flavonoids, quinones, saponins, sterols and tannins }\end{array}$ & $\begin{array}{c}{[94,95]} \\
{[96]}\end{array}$ \\
\hline M. hamata & $\begin{array}{l}\text { Whole plant/EtOH and } \mathrm{MeOH} \\
\text { extracts }\end{array}$ & $\begin{array}{c}\text { flavonoids, alkaloids, phytosterols, glycosides, tannins, phenolic compounds, } \\
\text { saponins and carbohydrates, }\end{array}$ & [97] \\
\hline M. invisa & Leaves/aq. extract & flavones, glycosides, saponins alkaloids and tannins & [98] \\
\hline M. albida & Whole plant/aq. extract & total phenolic contents & [99] \\
\hline M. scabrella bentham & Honeydew honeys & lignin-derived aldehydes, coumarins, phenolic acids and flavonoids & [100] \\
\hline M. rubicaulis & Stem/MeOH extract & flavonoids, tannins, triterpenes and carbohydrates & [101] \\
\hline
\end{tabular}

\subsection{Bioactive Constitutients of Genus Mimosa}

The plants of genus Mimosa are well known for their rich source of bioactive metabolites. Almost 145 active metabolites have been isolated from the genus Mimosa including chalcones, alkaloids, flavonoids, indoles, terpenes, terpenoids, saponins, steroids, amino acids, glycosides, flavanols, phenols, lignoids, polysaccharides, lignins, salts and fatty esters. This part of the paper documents the isolated secondary metabolites of the genus Mimosa in the past decades and their pharmaceutical values (Table 2). The structures of all the isolated compounds are presented in Figure 1. 
Table 2. Bioactive metabolites of genus Mimosa and their pharmacological potential.

\begin{tabular}{|c|c|c|c|c|c|c|c|}
\hline Species & Extract & Parts & Classification & Compounds & Modal/Assay & $\begin{array}{c}\text { Responses along with Critical } \\
\text { Assessment }\end{array}$ & Ref. \\
\hline \multirow{7}{*}{ M. tenuiflora } & DCM-Hex -MeOH & Stem bark & Chalcones & kukulkan A (1), kukulkan B (2) & & & [103] \\
\hline & Hex, ACE, MeOH & Leaves and flowers & Flavonoids & $\begin{array}{c}\text { 6-methoxy-4-O-metylnaringenin (3), } \\
\text { 6-methoxynaringenin (4), santin (5), } \\
\text { 4,5,7-trihydroxy-3,6-dimethoxy } \\
\text { flavone (6), 6-methoxykaempferol } \\
\text { (7), tenuiflorin A (8), tenuiflorin B } \\
\text { (9), tenuiflorin C (10), } \\
\text { 6-demethoxycapillarisin (11), } \\
\text { 6-demethoxy-4-O-methyl } \\
\text { capillarisin (12) }\end{array}$ & $\begin{array}{l}\text { In vitro/Antiprotozoal } \\
\text { assays/E. histolytica, } G \text {. } \\
\text { lamblia }\end{array}$ & $\begin{array}{c}\left(\mathrm{IC}_{50} \mu \mathrm{g} / \mathrm{mL}\right) \text { against } E . \text { histolytica } \text { and } \mathrm{G} \text {. } \\
\text { lamblia }(\mathbf{3})=72.7 \mu \mathrm{g} / \mathrm{mL} \text { and } 82.9 \\
\mu \mathrm{g} / \mathrm{mL} ;(\mathbf{4})=69.7 \mu \mathrm{g} / \mathrm{mL} \text { and } 75.3 \\
\mu \mathrm{g} / \mathrm{mL} ;(5)=76.4 \mu \mathrm{g} / \mathrm{mL} \text { and } 84.1 \\
\mu \mathrm{g} / \mathrm{mL} ;(\mathbf{6})=41.1 \mu \mathrm{g} / \mathrm{mL} \text { and } 108.6 \\
\mu \mathrm{g} / \mathrm{mL} ;(\mathbf{7})=69.8 \mu \mathrm{g} / \mathrm{mL} \text { and } 77.1 \\
\mu \mathrm{g} / \mathrm{mL} ;(8)=80.7 \mu \mathrm{g} / \mathrm{mL} \text { and } 91.8 \\
\mu \mathrm{g} / \mathrm{mL} ;(\mathbf{9})=71.6 \mu \mathrm{g} / \mathrm{mL} \text { and } 77.8 \\
\mu \mathrm{g} / \mathrm{mL} ;(\mathbf{1 0})=82.8 \mu \mathrm{g} / \mathrm{mL} \text { and } 92.8 \\
\mu \mathrm{g} / \mathrm{mL} ; \mathbf{( 1 1 )}=89.9 \mu \mathrm{g} / \mathrm{mL} \text { and } 100.9 \\
\mu \mathrm{g} / \mathrm{mL} ;(\mathbf{1 2})=78.7 \mu \mathrm{g} / \mathrm{mL} \text { and } 86.6 \\
\mu \mathrm{g} / \mathrm{mL} ; \text { positive control; emetinec }=2.2 \\
\mu \mathrm{g} / \mathrm{mL} \text { and } 0.8 \mu \mathrm{g} / \mathrm{mL} ; \text { Metronidazolec } \\
=0.23 \mu \mathrm{g} / \mathrm{mL} \text { and } 1.22 \mu \mathrm{g} / \mathrm{mL} \\
\text { respectively. Overall good activity }\end{array}$ & {$[104,105]$} \\
\hline & $\begin{array}{l}\text { MeOH, Crude } \\
\text { Alkaloid Extracts }\end{array}$ & Leaves and seeds & Indole alkaloid & $\begin{array}{c}N \text {-methyltryptamine (13), } \\
N, N \text {-dimethyltryptamine (14), } \\
\text { 2-methyltetrahydro- } \beta \text {-carboline (15) }\end{array}$ & & & [106] \\
\hline & Hex; ACE: $\mathrm{MeOH}$ & $\begin{array}{l}\text { Trunk bark, root } \\
\text { bark }\end{array}$ & Indole alkaloid & $\begin{array}{l}\mathrm{N}, \mathrm{N} \text {-dimethyltryptamine (14), } \\
\text { 5-hydroxy-tryptamine (16) }\end{array}$ & & & [107] \\
\hline & $\mathrm{MeOH}$ & Stem bark & $\begin{array}{l}\text { Terpenoids } \\
\text { saponins }\end{array}$ & $\begin{array}{l}\text { mimonoside A (17), mimonoside B } \\
(\mathbf{1 8}), \text { mimonoside C (19) }\end{array}$ & & & {$[13,108,109]$} \\
\hline & $\mathrm{MeOH}$ & Stem bark & Steroids saponins & $\begin{array}{c}\text { stigmasterol-3-O- } \beta \text {-D- } \\
\text { glucopyranosyl (20), } \beta \text {-sitosterol } \\
\text { 3-O- } \beta \text {-D-glucopyranosyl (21), lupeol } \\
\text { (22), campesterols (23), stigmasterol } \\
\text { (24), } \beta \text {-sitosterol (25), campesterol-3- } \\
\text { O- } \beta \text {-D-glucopyranosyl } \\
\text { (26) }\end{array}$ & & & [108] \\
\hline & $\mathrm{MeOH}$ & $\begin{array}{l}\text { Root bark, stem } \\
\text { bark }\end{array}$ & phytoindole Alkaloid & yuremamine (27) & & & {$[110,111]$} \\
\hline
\end{tabular}


Table 2. Cont.

\begin{tabular}{|c|c|c|c|c|c|c|c|}
\hline Species & Extract & Parts & Classification & Compounds & Modal/Assay & $\begin{array}{c}\text { Responses along with Critical } \\
\text { Assessment }\end{array}$ & Ref. \\
\hline \multirow[t]{3}{*}{ M. pigra } & $\begin{array}{l}\text { DEE and EtOAc } \\
\text { fraction }\end{array}$ & Leaves & $\begin{array}{l}\text { Acylated flavonol } \\
\text { glycosides }\end{array}$ & $\begin{array}{c}\text { myricetin (2-O-galloyl)- } \\
\text { 3-O- } \alpha \text {-L-rhamnopyranoside (28), } \\
\text { quercetin (2-O-galloyl)- } \\
\text { 3-O- } \alpha \text {-L-rhamnopyranoside (29), } \\
\text { myricetin } \\
\text { 3-O- } \alpha \text {-L-rhamnopyranoside (30), } \\
\text { quercetin } \\
\text { 3-O- } \alpha \text {-L-rhamnopyranoside (31), } \\
\text { quercetin } \\
\text { 3-O- } \beta \text {-L-arabinopyranoside (32) }\end{array}$ & & & [112] \\
\hline & $\mathrm{BuOH}$ & Stem bark & $\begin{array}{l}\text { Triterpene } \\
\text { glycosides }\end{array}$ & $\begin{array}{c}\text { Z/E-methoxycinnamic }(33,34), \\
\text { E-cinnamic acid }(35)\end{array}$ & & & [113] \\
\hline & $\begin{array}{l}\text { Hydro-MeOH } \\
\text { extract }\end{array}$ & Leaves & $\begin{array}{l}\text { Tryptophan, amino } \\
\text { acid and phenols }\end{array}$ & $\begin{array}{l}\text { tryptophan (36), myricitrin (37), } \\
\text { quercitrin (38), quercetin } \\
\text { 3-O-pentose (39), quercetin } \\
\text { 3-O-hexose (40), kampferol } \\
\text { 3-O-desoxyhexose (41) }\end{array}$ & & & [114] \\
\hline \multirow[t]{2}{*}{ M. caesalpiniifolia } & EtOH extract & Inflorescence & & $\begin{array}{c}\text { gallic acid (42), methylgallate (43), } \\
\text { 5-hydroxy-4,7-dimethoxy-flavone } \\
\text { (44), quercetin (38), } \\
\text { quercetin-O-hexoside (45), vicenin-2 } \\
\text { (46), rutin (47) }\end{array}$ & & & [115] \\
\hline & EtOH extract & leaves & $\begin{array}{l}\text { Phenols and } \\
\text { flavonids }\end{array}$ & $\begin{array}{c}\text { catechin (48), 2,3 } \\
\text { dihydroquercetagetin (49), } \\
\text { procyanidin (50) }\end{array}$ & & & [116] \\
\hline \multirow[t]{2}{*}{ M. hamata } & $\mathrm{MeOH}$ extract & Roots & $\begin{array}{l}\text { Triterpenoidal } \\
\text { Saponins }\end{array}$ & $\begin{array}{c}\text { mimonoside A (17), mimonoside B } \\
\text { (18), mimonoside C (19), saponin A; } \\
\text { (3-O-L rhamnopyran osyl } \\
\text {-D-glucopyranosylmorolic acid) (51), } \\
\text { saponin B; (3-O-L-Arabinosyl-D- } \\
\text { glucosylmorolic acid) } \\
\text { (52) }\end{array}$ & $\begin{array}{l}\text { In vitro/Antimicrobial } \\
\text { activity agar well } \\
\text { diffusion } \\
\text { method/against } B . \\
\text { subtilis, E. coli, } E \text {. } \\
\text { aerogenes, S. aureus, } P . \\
\text { aeruginosa, A. flavus, K. } \\
\text { pneumonia, A. niger, C. } \\
\text { albicans, R. bataticola } \\
\end{array}$ & $\begin{array}{c}\text { Good activity against Gram -ve bacteria } \\
\text { and fungi Marginal activity toward } \\
\text { Gram + ve bacteria }\end{array}$ & [117-119] \\
\hline & & & & & $\begin{array}{l}\text { Antioxidant } \\
\text { activity } / \text { DPPH free } \\
\text { radical scavenging } \\
\text { assay }\end{array}$ & $\begin{array}{c}\text { Compounds exhibited } \mathrm{IC}_{50} ;(\mathbf{1 7})=0.45 \\
\mu \mathrm{g} / \mathrm{mL} ;(\mathbf{1 8})=0.55 \mu \mathrm{g} / \mathrm{mL} ;(\mathbf{1 9})=0.60 \\
\mu \mathrm{g} / \mathrm{mL} ; \mathbf{( 5 1})=0.085 \mu \mathrm{g} / \mathrm{mL} ; \mathbf{( 5 2})=0.10 \\
\mu \mathrm{g} / \mathrm{mL} ; \text { Standard Quercetin }=0.06 \\
\mu \mathrm{g} / \mathrm{mL} \text {. Overall good activity }\end{array}$ & [119] \\
\hline
\end{tabular}


Table 2. Cont.

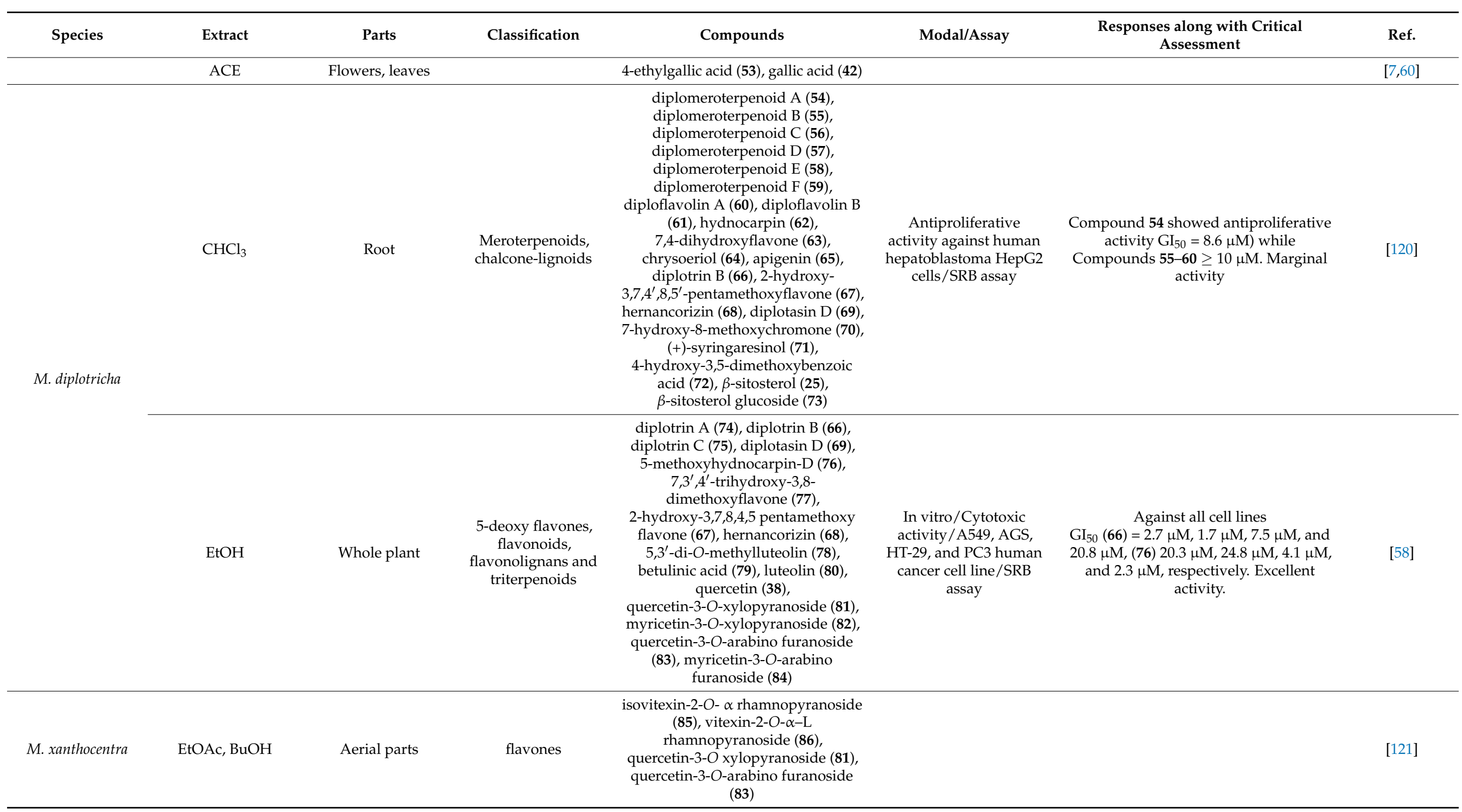


Table 2. Cont.

\begin{tabular}{|c|c|c|c|c|c|c|c|}
\hline Species & Extract & Parts & Classification & Compounds & Modal/Assay & $\begin{array}{c}\text { Responses along with Critical } \\
\text { Assessment }\end{array}$ & Ref. \\
\hline M. hostilis & EtOAc & Roots & Indole Alkaloid & $\mathrm{N}, \mathrm{N}$-dimethyltryptamine (14) & & & [122] \\
\hline M.artemisiana & $n$-Hex, MeOH & $\begin{array}{l}\text { Leaves and } \\
\text { branches }\end{array}$ & $\begin{array}{l}\text { Flavonoids, } \\
\text { flavonolignans, } \\
\text { glycosylated } \\
\text { steroid, triterpene, } \\
\text { steroids, indole } \\
\text { carboxylate }\end{array}$ & $\begin{array}{c}\text { quercetina-3-O-raminosídeo (87), } \\
\text { miricetina-3-O-raminoside (88), } \\
\text { Euphaline, } \\
\text { 3,5,4-trihydroxy-6,7-dimethoxy } \\
\text { flavone (89), flavonolignana(90,91), } \\
\text { sitosterol-3-O- } \beta \text {-D glycopyranoside } \\
\text { (21), lupeol (22), steroids sitostenone } \\
\text { (92), stigmastenone }(\mathbf{9 3}), \\
\text { campestenone }(\mathbf{9 4}), \text { campesterol (23), } \\
\text { stigmasterol (24), sitosterol (25), } \\
\text { methyl indole-3-carboxilate (95), } \\
\text { indole-3-carboxaldehyde (96) }\end{array}$ & & & [25] \\
\hline M. invisa & $\mathrm{DCM} / \mathrm{MeOH}$ & Aerial parts & Fatty aldol ester & $\begin{array}{l}\text { 17-O-triacontanoylheptadecanal (97) } \\
\text { and } \beta \text {-sitosterol (25), } \alpha \text {-amyrine (98), } \\
\text { lupeol (22), } \\
\text { 4-O-methylepinumisoflavone (99), } \\
\text { alpinumisoflavone (100), betulinic } \\
\text { acid (79), sitosterol } \\
\text { 3-O-B-D-glucopyranoside (21) and } \\
\text { epirobinetinidol (101) }\end{array}$ & $\begin{array}{l}\text { Antimicrobial } \\
\text { activity/E. coli, E. } \\
\text { aerogenes, S. aureus, } P . \\
\text { aeruginosa, K. pneumonia, } \\
\text { S. typhi, C. albicans/XTT } \\
\text { colorimetric assay }\end{array}$ & $\begin{array}{c}\text { Compound (97) and (101) were most } \\
\text { active against K. pneumonia } \\
\mathrm{MIC}=64 \mathrm{mg} / \mathrm{mL} \text {. Overall good activity }\end{array}$ & [28] \\
\hline \multirow[b]{2}{*}{ M. scabrella } & \multirow[b]{2}{*}{ Polysaccharide } & \multirow[b]{2}{*}{ Seeds } & & \multirow[b]{2}{*}{ Sulfated galactomannan (BRS) (102) } & $\begin{array}{l}\text { in vitro/antiviral } \\
\text { activity against Herpes } \\
\text { simplex virus } 1 \\
\text { (HSV-1)/plaque } \\
\text { reduction method }\end{array}$ & $\begin{array}{c}\text { At concentration } 20 \mu \mathrm{g} / \mathrm{mL} \\
\mathrm{IC}_{50} \text { lesser than } 2.5 \mu \mathrm{g} / \mathrm{mL} \text { was } \\
\text { observed. Marginal activity. Excellent } \\
\text { activity. }\end{array}$ & \multirow[b]{2}{*}{ [123] } \\
\hline & & & & & $\begin{array}{l}\text { in vitro/Cytotoxic } \\
\text { activity/in Vero and } \\
\text { MA-104 cells/MTT } \\
\text { assay }\end{array}$ & $\begin{array}{c}\text { At the concentrations } \geq 39 \mu \mathrm{g} / \mathrm{mL} \text { BRS } \\
\text { reduced by } 15 \% \text { the viability of Vero cells } \\
\qquad\left(C C_{50}=454 \mu \mathrm{g} / \mathrm{mL}\right) \\
\text { At the concentrations } 625 \mu \mathrm{g} / \mathrm{mL} \text { BRS } \\
\text { reduced by } 24 \% \text { the viability of MA-104 } \\
\text { cells }\left(C_{50}>625 \mu \mathrm{g} / \mathrm{mL}\right) \text {. Marginal } \\
\text { activity }\end{array}$ & \\
\hline M. somniam & $\mathrm{MeOH}$ & Whole plant & Alkaloid & $\begin{array}{l}\text { tryptamine (103), } N \text {-methyl } \\
\text { tryptamine (13) }\end{array}$ & & & [124] \\
\hline
\end{tabular}


Table 2. Cont.

\begin{tabular}{|c|c|c|c|c|c|c|c|}
\hline Species & Extract & Parts & Classification & Compounds & Modal/Assay & $\begin{array}{c}\text { Responses along with Critical } \\
\text { Assessment }\end{array}$ & Ref. \\
\hline \multirow{8}{*}{ M. pudica } & EtOAc fration & Whole plant & Flavonoid & $\begin{array}{c}\text { 2-(2',6'-dimethyl-3', } 4^{\prime}, 5^{\prime} \text {-alkyl or } \\
\text { hydroxy alkyl substituted } \\
\text { phenyl)-3-oxy-(alkyl or hydoxy } \\
\text { alkyl) 5,7-dihydroxy-chromen-4-one } \\
\text { (104-107) }\end{array}$ & $\begin{array}{c}\text { In vitro/Cytotoxic } \\
\text { activity/MTT } \\
\text { assay/human lung } \\
\text { adenocarcinoma cell } \\
\text { line (A549) \& human } \\
\text { erythroleukemic cell } \\
\text { line (K562) }\end{array}$ & $\begin{array}{c}\left(\mathrm{IC}_{50}\right) \text { of against } \mathrm{A} 549=76.67 \mu \mathrm{g} / \mathrm{mL} \\
\text { and } \mathrm{K} 562=287.63 \mu \mathrm{g} / \mathrm{mL}, \text { while } \\
\text { positive control Doxorubicin } \\
\mathrm{A} 549=2.76 \mu \mathrm{g} / \mathrm{mL} \text { and } \\
\mathrm{K} 562=4.72 \mu \mathrm{g} / \mathrm{mL} \text {. Marginal activity }\end{array}$ & [50] \\
\hline & HyOH extract & Whole plant & Amino acid & L-mimosine (108) & $\begin{array}{c}\text { Antioxidant } \\
\text { effect/DPPH radical } \\
\text { scavenging activity }\end{array}$ & $\begin{array}{c}\text { Compound at } 250 \mu \mathrm{g} / \mathrm{mL} \\
\left(\mathrm{IC}_{50}=233.06 \mu \mathrm{M}\right) . \text { Good activity }\end{array}$ & \multirow{2}{*}[34,125]{} \\
\hline & & & & & $\begin{array}{l}\text { In vitro/Cytotoxic } \\
\text { activity/daudi cell } \\
\text { line/MTT assay }\end{array}$ & $\begin{array}{c}\text { Compound 108, }\left(\mathrm{IC}_{50}=86.61 \mu \mathrm{M}\right) \text {. } \\
\text { Excellent activity }\end{array}$ & \\
\hline & $\mathrm{CHCl}_{3}$ extracts & Whole plant & Triterpenoid & triterpenoid glycoside (109) & & & [126] \\
\hline & $\mathrm{CHCl}_{3}$ extracts & Whole plant & - & $\begin{array}{l}\text { jasmonic acid (110), abscisic acid } \\
\text { (111), }\end{array}$ & & & [127] \\
\hline & $\mathrm{EtOH}$ & Whole plant & Flavonoids & $\begin{array}{c}5,7,3^{\prime}, 4^{\prime} \text {-tetrahydroxy-6-C-[ } \beta \text {-D- } \\
\text { apiose- }(1 \rightarrow 4)]-\beta \text {-D-glucopyranosyl } \\
\text { flavones }(\mathbf{1 1 2}), 7,8,3^{\prime}, 4^{\prime} \text {-tetrahydroxy- } \\
\text { 6-C-[ }[\alpha \text {-L-rhamnopyranosyl- }(1 \rightarrow 2)]- \\
\beta \text {-D-glucopyranosyl flavone }(\mathbf{1 1 3}), \\
5,7,4^{\prime} \text {-trihydroxyl-8-C- } \beta \text {-D- } \\
\text { glucopyranosyl flavones (114), } \\
\text { mimosinamine (115), mimosinic acid } \\
\text { (116), Tyrosin (117) }\end{array}$ & & & [128] \\
\hline & \multirow[t]{2}{*}{$\mathrm{EtOH}$} & Whole plant & Flavonoids & $\begin{array}{c}5,7,3^{\prime}, 4^{\prime} \text {-tetrahydroxy-6-C-[ } \beta \text {-D- } \\
\text { apiose-( }(1 \rightarrow 4)]-\beta \text {-D-glucopyranosyl } \\
\text { flavones }(\mathbf{1 1 2}), 6,7,3^{\prime}, 4^{\prime} \text {-tetrahydroxy- } \\
\text { 8-C-[ } \alpha \text {-L-rhamnopyranosyl- }(1 \rightarrow 2)]- \\
\beta \text {-D-glucopyranosyl flavone } \\
(\mathbf{1 1 8})\end{array}$ & & & [129] \\
\hline & & Arial parts & Flavonoids & $\begin{array}{c}\text { isoquercitrin (119), avicularin (120), } \\
\text { apigenin-7-O-D-glucoside (121), } \\
\text { cassiaoccidentalin B (122), orientin } \\
\text { (123), isoorientin (124) }\end{array}$ & & & [130] \\
\hline
\end{tabular}


Table 2. Cont.

\begin{tabular}{|c|c|c|c|c|c|c|c|}
\hline Species & Extract & Parts & Classification & Compounds & Modal/Assay & $\begin{array}{c}\text { Responses along with Critical } \\
\text { Assessment }\end{array}$ & Ref. \\
\hline \multirow{8}{*}{ M. pudica } & $\mathrm{MeOH}$ & Leaves & & chlorophyllin (125) & $\begin{array}{c}\text { Antimicrobial } \\
\text { activity/P. aeruginosa, E. } \\
\text { coli, S. aureus, K. } \\
\text { pneomoniae, C. } \\
\text { albicans/well diffusion } \\
\text { method }\end{array}$ & $\begin{array}{c}\text { Zone of inhibition at } 25 \mu \mathrm{g} / \mathrm{mL} \text { conc. } P \text {. } \\
\text { aeruginosa }=12 \mathrm{~mm}, \text { E. coli }=8 \mathrm{~mm}, S . \\
\text { aureus }=14 \mathrm{~mm}, \text { K. pneomoniae }=13 \mathrm{~mm} \text {, } \\
\text { C. albican }=9 \mathrm{~mm} . \text { At } 100 \mu \mathrm{g} / \mathrm{mL} \text { conc. } P \text {. } \\
\text { aeruginosa }=18 \mathrm{~mm}, \text { E. coli }=13 \mathrm{~mm}, S . \\
\text { aureus }=19 \mathrm{~mm}, K . \text { pneomoniae }=18 \mathrm{~mm}, \\
\text { C. albicans }=13 \mathrm{~mm} \text {. The streptomycin } \\
\text { sulphate and nystatin (standred) at } \\
10 \mu \mathrm{g} / \mathrm{mL} \text { showed maximum inhibition } \\
18 \mathrm{~mm}-19 \mathrm{~mm} . \\
\text { Good activity }\end{array}$ & [131] \\
\hline & $\begin{array}{l}\text { EtOAc-benzene } \\
\qquad(1: 9)\end{array}$ & Leaves & Phenolic ketone & $\begin{array}{l}\text { 4-(24'-methoxy-24'-methyl-1'-oxo- } \\
5^{\prime} \text {-n-propyl-tetracosanyl)- phenol } \\
(\mathbf{1 2 6})\end{array}$ & & & [132] \\
\hline & $\mathrm{EtOH}$ & Leaves & Flavonoids & $\begin{array}{c}\text { 7,3', } 4^{\prime} \text {-triacetoxy-3,8- } \\
\text { dimethoxyflavone }(\mathbf{1 2 7}), \\
\text { p-coumaric acid (128), } \\
7,3^{\prime}, 4^{\prime} \text {-trihydroxy-3,8- } \\
\text { dimethoxyflavone } \\
\text { (77) }\end{array}$ & & & [133] \\
\hline & $\mathrm{MeOH}$ & Leaves & & mimopudine (129) & & Responsible for leaves opening & [134] \\
\hline & $\mathrm{MeOH}$ & Leaves & & $\begin{array}{c}\text { potassium } \\
\text { 5-O- } \beta \text {-D-glucupyranosylgentisate } \\
\qquad(\mathbf{1 3 0})\end{array}$ & & Responsible for leaves closing & [135] \\
\hline & $\mathrm{MeOH}$ & Leaves & & $\begin{array}{l}\text { mimopudine (129), potassium } \\
\text { 5-O- } \beta \text {-D-glucupyranosylgentisate } \\
\text { (130), potassium L-malate (131), } \\
\text { magnesium potassium } \\
\text { trans-aconitate (132), dimethyl } \\
\text { ammoniumsalt (133) }\end{array}$ & & $\begin{array}{l}\text { Responsible for rapid sensitive actions, } \\
\text { such as heat and touch. } \\
\text { Periodic slow actions, such as nyctinastic } \\
\text { actions }\end{array}$ & [136] \\
\hline & & Fresh leaves & & tubulin (134) & & & [137] \\
\hline & EtOH & Leaves & & $\begin{array}{l}\text { nor-epinephrine (135), d-pinitol } \\
\text { (136), } \beta \text {-sitosterol (25) }\end{array}$ & & & [138] \\
\hline
\end{tabular}


Table 2. Cont.

\begin{tabular}{|c|c|c|c|c|c|c|c|}
\hline Species & Extract & Parts & Classification & Compounds & Modal/Assay & $\begin{array}{c}\text { Responses along with Critical } \\
\text { Assessment }\end{array}$ & Ref. \\
\hline & $\mathrm{EtOH}$ & Leaves & & $\begin{array}{c}5,7,3^{\prime}, 4^{\prime} \text {-tetrahydroxy-6-C-[ } \beta \text {-D- } \\
\text { apiose- }(1 \rightarrow 4)]-\beta \text {-D-glycopyranosyl } \\
\text { flavone }(\mathbf{1 1 2}), \text { orientin }(\mathbf{1 2 3}), \\
\text { isorientin }(\mathbf{1 2 4}) \\
\text { vitexin }(\mathbf{1 3 7}), \text { isovitexin }(\mathbf{1 3 8})\end{array}$ & & & [139] \\
\hline & & Roots & Chroman & $\begin{array}{l}\text { 2-hydroxymethyl-chroman-4-one } \\
\text { (139) }\end{array}$ & $\begin{array}{c}\text { Antifungal } \\
\text { activity/dilution agar } \\
\text { plate method } / P \text {. } \\
\text { ultimum, } P \text {. capsici, } R \text {. } \\
\text { solani, B. cinerea, } A \text {. } \\
\text { panax and } S \text {. sclerotiorum }\end{array}$ & $\begin{array}{c}\text { Compound (139) showed good } \mathrm{ED}_{50} \\
\text { value against } \\
\text { P. capsici }=35.7 \mu \mathrm{g} / \mathrm{mL} \\
\text { S. sclerotiorum }=52.1 \mu \mathrm{g} / \mathrm{mL} \\
\text { P. ultimum }=54.9 \mu \mathrm{g} / \mathrm{mL} \\
\text { Overall good activity }\end{array}$ & [140] \\
\hline & & Roots & Sterolglucoside & $\begin{array}{c}\text { stigmasterol (24), } \beta \text {-sitosterol (25), } \\
\text { betulinic acid (79), } \\
\text { 4-a,24-dimethylcholest-7-en-3 } \beta \text {-ol- } \\
\text { 3 } \beta \text {-D-glucoside } \\
\text { (140) }\end{array}$ & & & [141] \\
\hline & $\mathrm{MeOH}$ & Roots & Diterpenoids & $\begin{array}{c}\text { 19-O-trans-feruloyl-labd-8(17)-en- } \\
\text { 15,19-diol (141), } \\
\text { 19-O-[(E)-3', } 4^{\prime} \text {-dimethoxy } \\
\text { cinnamoyl]-labd-8(17)-en- } 15,19 \text {-diol } \\
(\mathbf{1 4 2})\end{array}$ & & & [142] \\
\hline & & Seeds & Fatty acids & $\begin{array}{l}\text { D-xylose (143), D-glucuronic acid } \\
\text { 4-O-(3,5-dihydroxybenzoic } \\
\text { acid)-b-D-glucuronide (144) }\end{array}$ & & & [143] \\
\hline & & Seeds & Cardiac glycosides & bufadienolide (145) & & & [144] \\
\hline & & Stem & Amino acids & mimosine (108) & & & [145] \\
\hline
\end{tabular}


<smiles>[R]c1ccc(/C=C/C(=O)c2ccc(O)c(OC)c2O)cc1</smiles>

(1) $\mathrm{R}=\mathrm{OCH}_{3}$

(2) $\mathrm{R}=\mathrm{OH}$<smiles>[R20]c1ccc(Oc2cc(=O)c3c(O)c([R])c(O)cc3o2)cc1[R]</smiles>

(8) $\mathrm{R}=\mathrm{OCH}_{3} ; \mathrm{R} 1=\mathrm{OH} ; \mathrm{R}_{2}=\mathrm{CH}_{3}$

(9) $\mathrm{R}, \mathrm{R}_{1}=\mathrm{OCH}_{3} ; \mathrm{R}_{2}=\mathrm{H}$

(10) $\mathrm{R}=\mathrm{H} ; \mathrm{R}_{1}=\mathrm{OH} ; \mathrm{R}_{2}=\mathrm{CH}_{3}$

(11) $R, R_{1}, R_{2}=H$

(12) $\mathrm{R}, \mathrm{R}_{1}=\mathrm{H} ; \mathrm{R}_{2}=\mathrm{CH}_{3}$<smiles>COC1=CC(c2ccc(O)cc2)Oc2cc(O)c(OC)c(O)c21</smiles>

(3)<smiles>[R]N(C)CCc1c[nH]c2ccccc12</smiles>

(13) $\mathrm{R}=\mathrm{H}$ (14) $\mathrm{R}=\mathrm{CH}_{3}$<smiles>COc1c(O)cc2c(c1O)C(=O)C[C@@H](c1ccc(O)cc1)O2</smiles>

(4)<smiles>CN1CCc2c([nH]c3ccccc23)C1</smiles>

(15)<smiles>[R]c1ccc(-c2oc3cc(O)c(OC)c(O)c3c(=O)c2[R])cc1</smiles>

(5) $\mathrm{R}=\mathrm{OCH}_{3} ; \mathrm{R}_{1}=\mathrm{OCH}_{3}$ (6) $\mathrm{R}=\mathrm{OH} ; \mathrm{R}_{1}=\mathrm{OCH}_{3}$ (7) $\mathrm{R}=\mathrm{OH} ; \mathrm{R}_{1}=\mathrm{OH}$

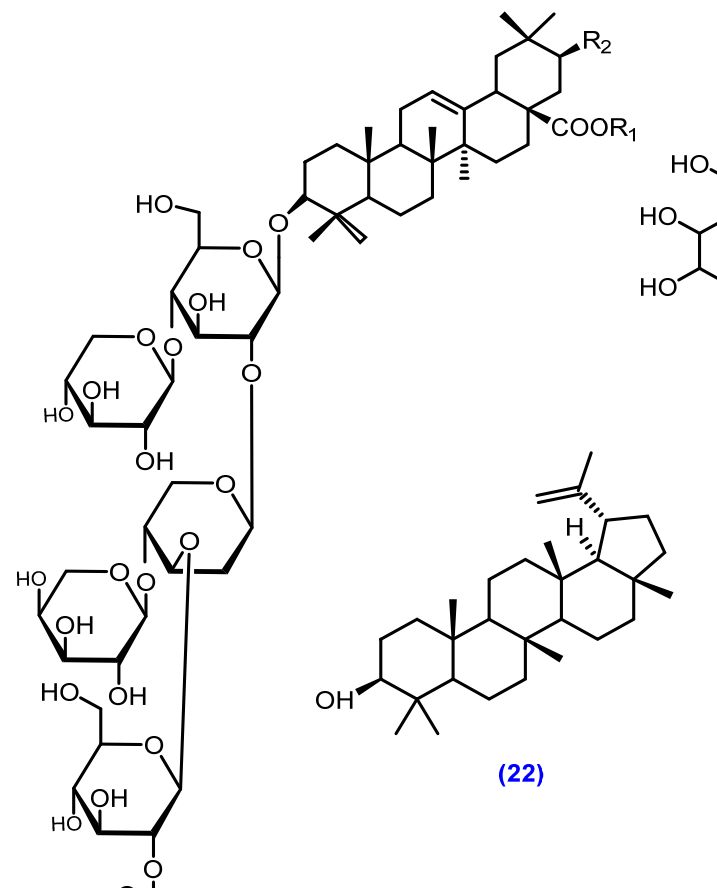

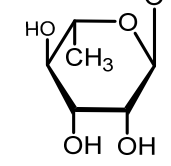

(17) $\mathrm{R}_{1}=\underbrace{\mathrm{OH}_{3}}_{\mathrm{OH} O H} ; \mathrm{R}_{2}=\mathrm{H}$

(18) $\mathrm{R}_{1}, \mathrm{R}_{2}=\mathrm{H}$

(19) $\mathrm{R}_{1}=\underbrace{\mathrm{OH}}_{\mathrm{OH}} ; \mathrm{R}_{2}=\mathrm{OH}$<smiles>CN(C)CCc1c2n(c3ccccc13)[C@@H](c1cc(O)c(O)c(O)c1)C(O)[C@H]2c1ccc(O)cc1O</smiles>

(27)<smiles>[R]C1CCC2C(CCC(C)CCC(C)C(C)CCC(C=CC(C)C)C(C)C)C1CCC1C2CC=C2CC(O)CCC21C</smiles>

(21)<smiles>CC(C)C(C)CCC(C)C1CCC2C3CC=C4CC(OC5O[C@H](CO)C(O)C(O)C5O)CC[C@]4(C)C3CCC12C</smiles><smiles>[R]c1cc(-c2oc3cc(O)cc(O)c3c(=O)c2OC2O[C@H](C)[C@@H](O)[C@H](O)[C@H]2OC(=O)c2cc(O)c(O)c(O)c2)cc(O)c1O</smiles>

(28) $\mathrm{R}=\mathrm{OH}$

(29) $\mathrm{R}=\mathrm{H}$

Figure 1. Cont. 
<smiles>[R]c1cc(-c2oc3cc(O)cc(O)c3c2O[C@H]2OC(Br)[C@H](O)[C@@H](O)[C@H]2O)cc(O)c1O</smiles>

(30) $\mathrm{R}=\mathrm{OH} ; \mathrm{R}_{1}=\mathrm{CH}_{3}$ (31) $\mathrm{R}=\mathrm{H} ; \mathrm{R}_{1}=\mathrm{CH}_{3}$ (32) $\mathrm{R}=\mathrm{H} ; \mathrm{R}_{1}=\mathrm{H}$<smiles>[R1]Oc1c(-c2cc([R2])c(O)c(O)c2)oc2cc(O)cc(O)c2c1=O</smiles>

(37) $\mathrm{R}_{1}=\mathrm{H} ; \mathrm{R}_{2}=\mathrm{OH}$

(38) $\mathrm{R}_{1}=\mathrm{H} ; \mathrm{R}_{2}=\mathrm{H}$

(39) $R_{1}=$ pentose; $R_{2}=H$

(40) $R_{1}=$ hexose; $R_{2}=H$<smiles>O=C(OCCOC(=O)Oc1c(-c2ccc(O)cc2)oc2cc(O)cc(O)c2c1=O)OCc1ccccc1</smiles>

(41)<smiles>O=c1c(OC2OC(CO)[C@@H](O)[C@H](O)C2O)c(-c2ccc(O)c(O)c2)oc2cc(O)cc(O)c12</smiles>

(45)<smiles>N[C@@H](Cc1c[nH]c2ccccc12)C(=O)O</smiles>

(36)

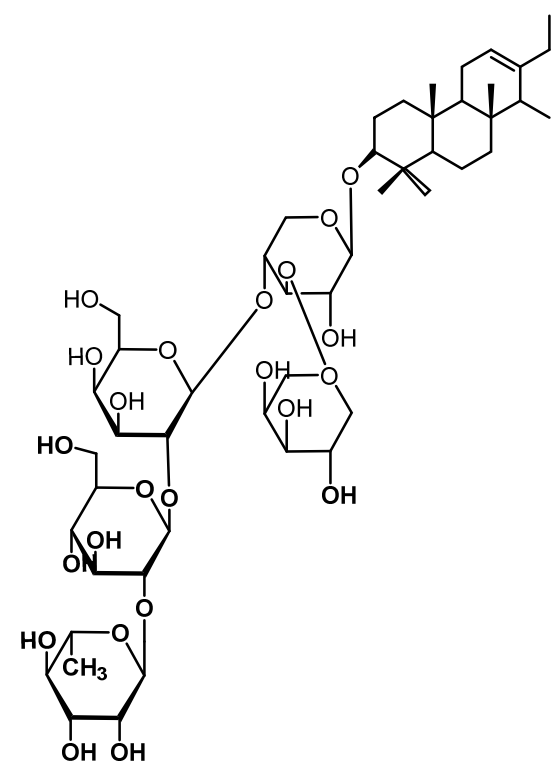

(33) $\mathrm{R}=-\mathrm{OCHO}-\mathrm{CH}=\mathrm{CH} \longrightarrow-\mathrm{OCH}_{3}$

(34) $\mathrm{R}=\mathrm{H}$

(35) $\mathrm{R}=-\mathrm{OCHO}-\mathrm{CH}=\mathrm{CH} \longrightarrow$<smiles>COc1ccc(-c2cc(=O)c3c(O)cc(OC)cc3o2)cc1</smiles>

(44)<smiles>CC1OC(OCC2CC(OC3=C(c4ccc(O)c(O)c4)Cc4cc(O)cc(O)c4C3=O)C(O)C(O)C2O)C(O)C(O)C1O</smiles>

(47)

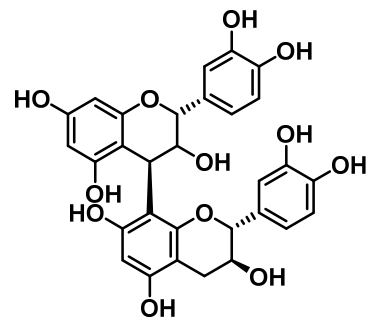

(50)

Figure 1. Cont. 
<smiles>[R6]C1CCC2(C)C3CCC4C5=CC(C)(C)CCC5(C(=O)O)CCC4(C)C3CCC2(C)C1(C)C</smiles>

$R=$ Glu-Rha

(52) $R=$ Ara-Glu<smiles>[R]c1cc(O)cc2oc([C@]3(C)C(=O)C=C(OC)C(=O)[C@@H]3CC=C(C)C)cc(=O)c12</smiles><smiles>O=C(/C=C/c1ccc2c(c1)C(CO)[C@H](c1ccc(O)cc1)O2)c1ccc(O)cc1O</smiles>

(60)<smiles>CCOC(=O)c1cc(O)c(O)c(O)c1</smiles>

(53)

(58) $\mathbf{R}=\mathrm{OH}$

(59) $\mathrm{R}=\mathrm{H}$<smiles>COc1cc([C@H]2Oc3ccc(C4=COc5c(O)cc(O)cc5O4)cc3OC2CO)ccc1O</smiles>

(62)<smiles>COc1cc(-c2oc3c(OC)c(OC)ccc3c(=O)c2OC)c(OC)cc1O</smiles>

(67)<smiles>[R2]c1cc(-c2cc(=O)c3c([R])cc(O)cc3o2)ccc1O</smiles>

(63) $R_{1}=R_{2}=H$

(64) $\mathrm{R}_{1}=\mathrm{OH}, \mathrm{R}_{2}=\mathrm{OCH}_{3}$

(65) $\mathrm{R}_{1}=\mathrm{OH}, \mathrm{R}_{2}=\mathrm{H}$<smiles>COc1cc(O)c2c(=O)cc(-c3cc(OC)c(OC)cc3O)oc2c1</smiles>

(68)

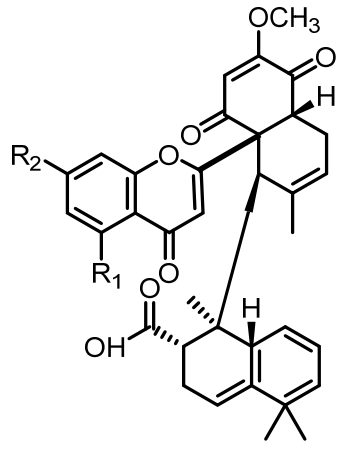

(54) $\mathrm{R}_{1}=\mathrm{OH}, \mathrm{R}_{2}=\mathrm{OCH}_{3}$

(55) $\mathrm{R}_{1}=\mathrm{OH}, \mathrm{R}_{2}=\mathrm{OH}$

(56) $\mathrm{R}_{1}=\mathrm{H}, \mathrm{R}_{2}=\mathrm{OCH}_{3}$

(57) $\mathrm{R}_{1}=\mathrm{H}, \mathrm{R}_{2}=\mathrm{OH}$<smiles>COc1cc([C@H]2Oc3ccc(/C=C/C(=O)c4ccc(O)cc4O)cc3O[C@H]2CO)ccc1O</smiles>

(61)

(66)<smiles>COc1ccc2c(c1O)COc1c-2oc2c(OC)c(OC)ccc2c1=O</smiles>

(69)

Figure 1. Cont. 


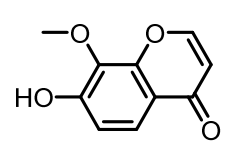

(70)<smiles>COc1cc([C@@H]2OC[C@H]3[C@H]2CO[C@H]3c2cc(OC)c(O)c(OC)c2)cc(OC)c1O</smiles>

(71)<smiles>COc1cc(C(=O)O)cc(OC)c1O</smiles>

(72)<smiles>CCC(CCC(C)C1CCC2C3CC=C4CC(OC5OC(CO)C(O)C(O)C5O)CCC4(C)C3CCC12C)C(C)C</smiles>

(73)<smiles>COc1c(-c2ccc(O)c(O)c2)oc2c(OC)c(O)ccc2c1=O</smiles>

(77)

(80)<smiles>O=c1cc(-c2ccc(O)c(O)c2)oc2cc(O)cc(O)c12</smiles><smiles>[R]c1cc([R])c(-c2oc3c([R])c([R])ccc3c(=O)c2[R])cc1[R6]</smiles>

(74) $\mathrm{R}_{1}=\mathrm{R}_{2}=\mathrm{R}_{3}=\mathrm{R}_{5}=\mathrm{OCH}_{3}, \mathrm{R}_{4}=\mathrm{R}_{6}=\mathrm{OH}$ (75) $\mathrm{R}_{2}=\mathrm{R}_{5}=\mathrm{R}_{6}=\mathrm{OCH}_{3}, \mathrm{R}_{4}=\mathrm{OH}, \mathrm{R}_{1}=\mathrm{R}_{3}=\mathrm{H}$<smiles>COc1cc(-c2cc(=O)c3c(OC)cc(O)cc3o2)ccc1O</smiles>

(78)<smiles>COc1cc([C@H]2Oc3cc(C4CC(=O)c5c(O)cc(O)cc5O4)ccc3O[C@H]2CO)cc(OC)c1O</smiles>

(76)

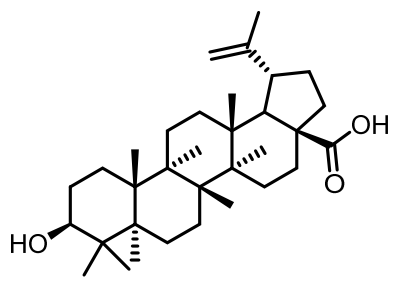

(79)<smiles>[R]c1cc(-c2oc3cc(O)cc(O)c3c(=O)c2OC2OC[C@@H](O)[C@H](O)[C@H]2O)cc(O)c1O</smiles>

(81) $\mathrm{R}=\mathrm{H}$

(82) $\mathrm{R}=\mathrm{OH}$<smiles>[R]c1cc(-c2oc3cc(O)cc(O)c3c(=O)c2O[C@@H]2O[C@H](CO)[C@@H](O)[C@H]2O)cc(O)c1O</smiles>

$\mathrm{R}=\mathrm{H}$

(84) $\mathrm{R}=\mathrm{OH}$

Figure 1. Cont. 
<smiles>C[C@@H]1O[C@H](O[C@H]2[C@@H](c3c(O)cc4c(c3O)C(=O)CC(c3ccc(O)cc3)O4)OC(CO)[C@@H](O)[C@H]2O)[C@H](O)[C@@H](O)[C@H]1O</smiles>

(85)<smiles>COc1cc2oc(-c3ccc(O)cc3)c(O)c(=O)c2c(O)c1OC</smiles>

(89)<smiles>C[C@H]1O[C@H](O[C@H]2C(O)[C@H](O)C(CO)O[C@H]2c2c(O)cc(O)c3c2OC(c2ccc(O)cc2)CC3=O)[C@H](O)[C@@H](O)[C@H]1O</smiles>

(86)<smiles>[R]c1cc(COc2ccc(C3Oc4cc(O)cc(O)c4C(=O)C3[R3])cc2OCCO)cc(OC)c1[R]</smiles>

(90) $\mathrm{R}_{1}=\mathrm{OH}, \mathrm{R}_{2}=\mathrm{OCH}_{3}, \mathrm{R}_{3}=\mathrm{H}$ (91) $R_{1}=O_{C H}, R_{2}=H, R_{3}=O H$

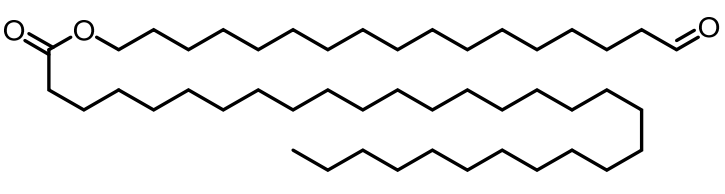

(97)<smiles>CC1(C)C=Cc2c(cc3occ(-c4ccc(O)cc4)c(=O)c3c2O)O1</smiles>

(100)<smiles>[R]c1cc(-c2oc3cc(O)cc(O)c3c(=O)c2O[C@@H]2O[C@H](C)[C@@H](O)[C@H](O)[C@H]2O)cc(O)c1O</smiles>

(87) $\mathrm{R}=\mathrm{H}$

(88) $\mathrm{R}=\mathrm{OH}$<smiles>[R]C1CCC2CC3CCC4=CC(=O)CC[C@]4(C)C3CC12</smiles>

(92) $\mathrm{R}=(\mathrm{a})$

(93) $R=$ (b)

(94) $R=(c)$
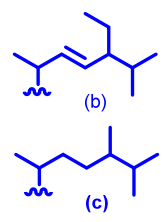

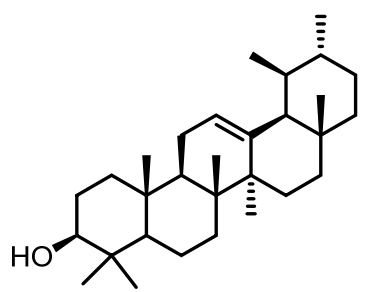

(98)<smiles>Oc1ccc2c(c1)O[C@H](c1cc(O)c(O)c(O)c1)C(O)C2</smiles>

(101)

Figure 1. Cont. 

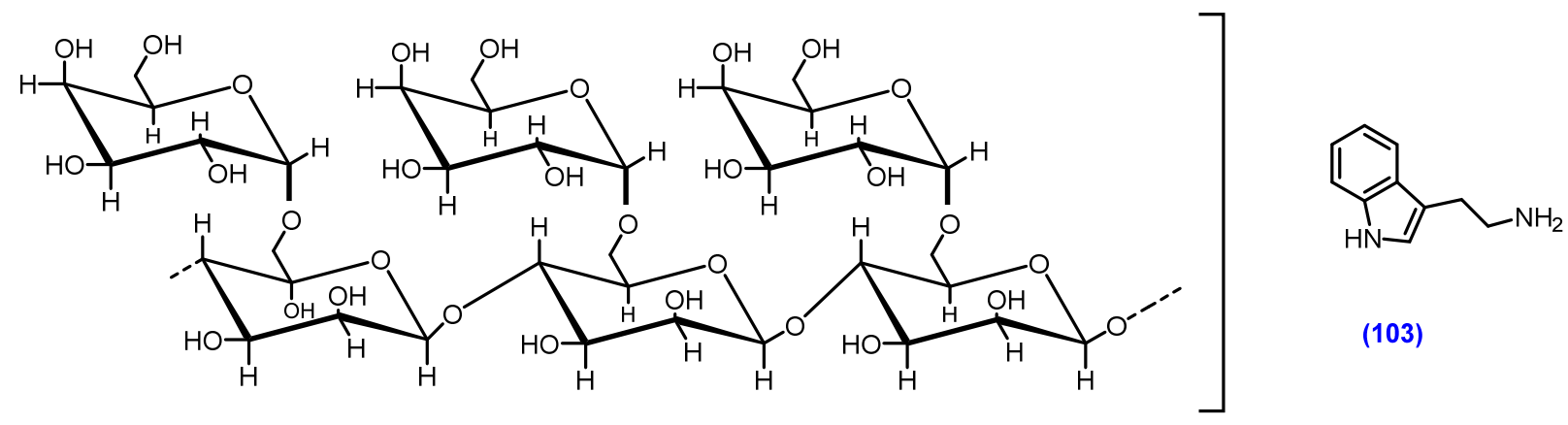

(102)

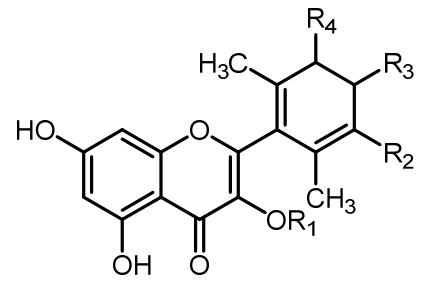

(104) $-\mathrm{O}-\mathrm{CH}=\mathrm{CH}-\mathrm{O}-\mathrm{CH}=\mathrm{CH}-\mathrm{OH}$

(105) $-\mathrm{O}-\mathrm{CH}=\mathrm{CH}-\mathrm{CH}=\mathrm{CH}-\mathrm{CH}=\mathrm{CH}-\mathrm{CH}_{3}$

(106) $-\mathrm{O}-\mathrm{CH}=\mathrm{CH}-\mathrm{CH}=\mathrm{CH}-\mathrm{CH}=\mathrm{CH}_{2}$

(107) $-\mathrm{CH}=\mathrm{CH}-\mathrm{CH}=\mathrm{CH}_{2}$<smiles>N[C@H](Cn1ccc(=O)c(O)c1)C(=O)O</smiles>

(108)

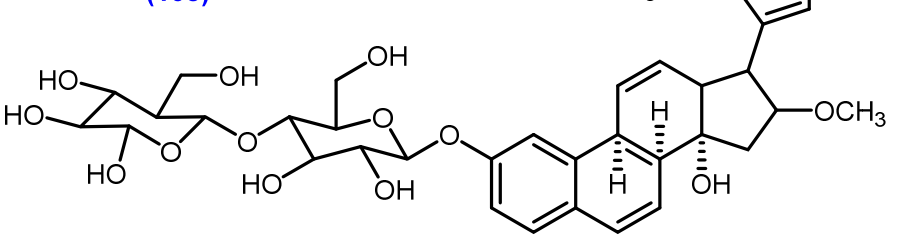

(109)<smiles>CC/C=C\C[C@H]1C(=O)CC[C@@H]1CC(=O)O</smiles>

(110)<smiles>O=c1cc(-c2ccccc2)oc2c(O)c(O)c(Cl)cc12</smiles>

(113)<smiles>CC1=CC(=O)CC(C)(C)[C@]1(O)/C=C/C(C)=C\C(=O)O</smiles><smiles>OCCC1C(O)C(O)C2OC(C1O)C(Oc1c(O)cc(O)cc1O)C(O)C2O</smiles>

(114)<smiles>O=c1cc(-c2ccc(O)c(O)c2)oc2cc(O)c(Cl)c(O)c12</smiles>

(112)<smiles>NNC(Cn1ccc(=O)c(O)c1)C(=O)O</smiles>

(115)

Figure 1. Cont. 
<smiles>NC(Cn1ccc(=O)c(O)c1)C(=O)O</smiles>

(116)<smiles>NC(Cc1ccc(O)cc1)C(=O)O</smiles>

(117)

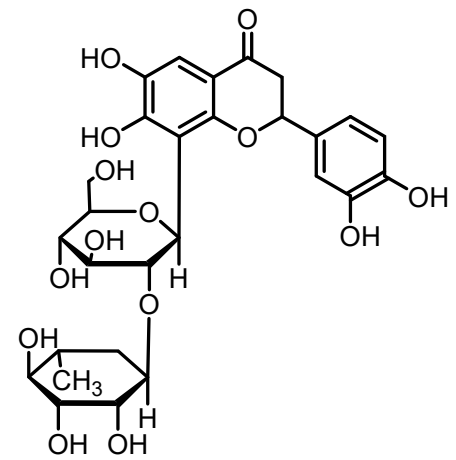

(118)<smiles>O=c1c(OC2OC(CO)[C@@H](O)[C@H](O)[C@H]2O)c(-c2ccc(O)c(O)c2)oc2cc(O)cc(O)c12</smiles>

(119)<smiles>O=c1c(OC2O[C@H](CO)C(O)[C@H]2O)c(-c2ccc(O)c(O)c2)oc2cc(O)cc(O)c12</smiles>

(120)<smiles>O=c1cc(-c2ccc(O)cc2)oc2cc(O[C@@H]3O[C@H](CO)[C@@H](O)[C@H](O)[C@H]3O)cc(O)c12</smiles><smiles>C[C@@H]1O[C@H](c2c(O)cc3oc(-c4ccc(O)c(O)c4)cc(=O)c3c2O)[C@H](OC2O[C@H](C)C(O)[C@H](O)[C@H]2O)C(=O)[C@H](O)[C@@H]1O</smiles>

(122)

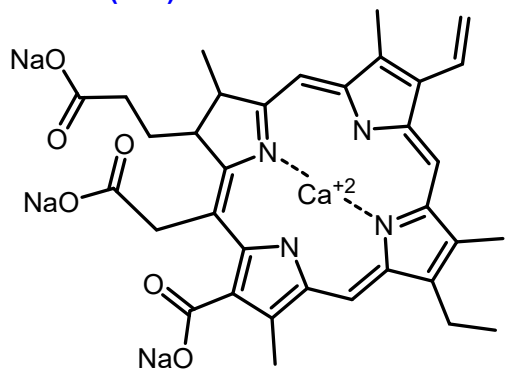

(125)<smiles>O=c1cc(-c2ccc(O)c(O)c2)oc2c([C@H]3OC(CO)[C@H](O)[C@H](O)[C@H]3O)c(O)cc(O)c12</smiles>

(123)<smiles>CCC(C)C(CCCCC(=O)c1ccc(O)cc1)CCCC(C)OC</smiles><smiles>COc1ccc(-c2oc3c(OC)c(OC(C)=O)ccc3c(=O)c2OC)cc1OC(C)=O</smiles>

(127)

Figure 1. Cont. 
<smiles>O=C(O)/C=C/c1ccc(O)cc1</smiles>

(128)<smiles>[14CH3]C(=O)CC(O)C(=O)[OH2+]</smiles>

(131)<smiles>NC(C(O)N(O)C(O)C(N)n1ccc(=O)cc1)n1ccc(=O)cc1</smiles>

(129)<smiles>O=C(O[Na])c1cc(OC2OC(CO)C3(CO)OC2C(O)C(O)C3O)ccc1O</smiles>

(130)<smiles>C[NH2+]C</smiles>

(133)<smiles>COc1ccc(C=C2c3ccccc3C(=O)c3ccccc32)cc1O</smiles>

(134)<smiles>NCC(O)c1ccc(O)c(O)c1</smiles>

(135)<smiles>COC1C(O)C(O)C(O)[C@@H](O)C1O</smiles>

(136)<smiles>[14CH3]C(=O)C(=CC(=O)[O-])C(=O)[O+]</smiles>

(132)<smiles>O=c1cc(-c2ccc(O)cc2)oc2c(C3OC(CO)[C@H](O)[C@H](O)[C@H]3O)c(O)cc(O)c12</smiles><smiles>O=c1cc(-c2ccc(O)cc2)oc2cc(O)c(C3O[C@H](CO)[C@@H](O)[C@H](O)[C@H]3O)c(O)c12</smiles>

(138)<smiles>O=C1CC(CO)Oc2ccccc21</smiles>

(139)<smiles>CC(C)C(C)CC[C@H](C)C1CCC2C3=CCC4[C@@H](C)C(OC5C[C@H](O)[C@@H](O)C(CO)O5)CC[C@]4(C)C3CCC21C</smiles>

(140)<smiles>[R]Oc1ccc(/C=C/C(=O)OC[C@]2(C)CCC[C@]3(C)[C@H](CCC(C)C)/C(=C\[C@@H](C)CCO)CC[C@H]23)cc1OC</smiles>

(141) $\mathrm{R}=\mathrm{H}$ (142) $\mathrm{R}=\mathrm{CH}_{3}$<smiles>OC1OC[C@@H](O)[C@H](O)[C@H]1O</smiles>

(143)<smiles>CCCCCCCCCCCCCCCCCC(=O)O</smiles>

(144)<smiles>C[C@]12CC[C@H](c3ccc(=O)oc3)CC[C@@H]1[C@@H]1CCC3CCCC[C@@H]3[C@@H]12</smiles>

(145)

Figure 1. Bioactive constituents isolated from various species of genus Mimosa. 


\subsubsection{M. tenuiflora}

Dominguez et al. isolated two chalcones called kukulkan A (2', $4^{\prime}$-dihydroxy- $3^{\prime}, 4-$ dimetoxy chalcone) (1) and kukulkan B $\left(2^{\prime}, 4^{\prime}, 4\right.$ - trihydroxy-3'-metoxychalcone) (2) from $M$. tenuiflora stem bark in the form of yellow crystals [103]. Ten different flavonoids including the chromones named 6-methoxy-4-O-metylnaringenin (3), 6-methoxy naringenin (4), santin (5), 4,5,7-trihydroxy-3,6-dimethoxy flavone (6), 6-methoxykaempferol (7), tenuiflorin A [5,7-dihydroxy-2-(3-hydroxy-4-methoxy phenoxy)-6-methoxychromone] (8), tenuiflorin B [5,7-dihydroxy-2-(4-hydroxy-3-methoxy phenoxy)-6-methoxychromone] (9), tenuiflorin C [5,7-dihydroxy-2-(3-hydroxy-4-methoxy phenoxy)-chromone] (10), 6-demethoxycapillarisin (11) and 6-demethoxy-4-O-methylcapillarisin (12) were isolated from the flowers and leaves of M. tenuiflora [104,105]. Gardner et al. reported on the alkaloids $N$-methyl-tryptamine (13), $N, N$-dimethyltryptamine (14) and 2-methyltetrahydro- $\beta$-carboline (15) from the $\mathrm{MeOH}$ and crude extracts of leaves and seeds of M. tenuiflora [106]. Meckes-Lozoya et al. isolated the alkolides 5-hydroxy-tryptamine (16) and $N, N$-dimethyl tryptamine (14) from the Hex:ACE:MeOH extracts of root bark of $M$. tenuiflora [107]. Different terpenoidal saponins called mimonoside A (17), mimonoside B (18) and mimonoside C (19); steroids saponins called stigmasterol-3-O- $\beta$-D-glucopyranosyl (20), $\beta$-sitosterol-3-O- $\beta$-D-glucopyranosyl (21), lupeol (22), campesterols (23), stigmasterol (24), $\beta$-sitosterol (25) and campesterol-3-O$\beta$-D-glucopyranosyl (26) were isolated from the $\mathrm{MeOH}$ extract of the stem bark of $M$. tenuiflora $[13,108,109]$. The phytoindole alkaloid yuremamine (27) was isolated from the $\mathrm{MeOH}$ extract of $M$. tenuiflora leaves [110,111].

\subsubsection{M. pigra}

The novel acylated flavanol glycosides myricetin (2-O-galloyl)-3-O- $\alpha$-L-rhamnopyranoside (28), quercetin(2-O-galloyl)-3-O- $\alpha$-L-rhamnopyranoside (29), myricetin 3-O- $\alpha$-L-rhamno pyranoside (30), quercetin $3-O-\alpha$-L-rhamnopyranoside (31) and quercetin $3-O-\beta$-L-arabino pyranoside (32) were isolated from $M$. pigra leaves [112]. Englert et al. isolated two novel triterpene glycosides called Z/E-methoxycinnamic $(33,34)$ or an E-cinnamic acid (35) from the $\mathrm{BuOH}$ extract of $M$. pigra stem bark [113]. Rakotomalala et al. reported on the isolation of tryptophan (36), myricitrin (37), quercitrin (38), quercetin 3-O-hexose (39), quercetin 3-O-pentose (40) and kampferol 3-O-desoxyhexose (41) from the hydro-MeOH extract of M. pigra leaves [114].

\subsubsection{M. caesalpiniifolia}

Santos et al. isolated gallic acid (42), methylgallate (43), 5-hydroxy-4,7-dimethoxyflavone (44), quercetin (38), quercetin-O-hexoside (45), vicenin-2 (46) and rutin (47) from the EtOH extract of inflorescence of $M$. caesalpiniifolia [115]. Silva et al. isolated the phenolic compounds called catechin (48), 2,3 dihydroquercetagetin (49) and procyanidin (50) from the EtOH extract of M. caesalpiniifolia leaves [116].

\subsubsection{M. hamata}

The compounds mimonoside A (17), mimonoside B (18), mimonoside C (19), saponin A (51) and saponin B (52) were isolated from the leaves and roots of $M$. hamata [117-119]. Mehta et al. isolated 4-ethylgallic acid (53) from the ACE extract of the flowers of $M$. hamata [60], while gallic acid (42) and 4- ethylgallic acid (53) were found in the leaves of $M$. hamata [7].

\subsubsection{M. diplotricha}

Chiou et al. isolated six new meroterpenoids called diplomeroterpenoids A-F (5459), two new chalcone-lignoids called diplochalcolins $A$ and $B(\mathbf{6 0 , 6 1 )}$ and 13 known compounds called hydnocarpin (62), 7,4-dihydroxyflavone (63), chrysoeriol (64), apigenin (65), diplotrin B (66), 2-hydroxy-3,7,4', 8, $5^{\prime}$-pentamethoxyflavone (67), hernancorizin (68), diplotasin D (69), 7-hydroxy-8-methoxychromone (70), (+)-syringaresinol (71), 4-hydroxy3,5 -dimethoxybenzoic acid (72), $\beta$-sitosterol (25), $\beta$-sitosterol glucoside (73) from $\mathrm{CHCl}_{3}$ 
extract of $M$. diplotricha roots [120]. Lin et al. reported four 5-deoxyflavones called diplotrin A (74), diplotrin B (66), diplotrin C (75), diplotasin D (69), 5-methoxyhydnocarpin-D (76), 7,3', '4'-trihydroxy-3,8-dimethoxyflavone (77), 2-hydroxy-3,7,8,4,5-pentamethoxyflavone (67), hernancorizin (68), 5,3'-di-O-methylluteolin (78), betulinic acid (79), luteolin (80), quercetin (38), quercetin-3-O-xylopyranoside (81), myricetin-3-O-xylopyranoside (82), quercetin-3-Oarabino furanoside (83) and myricetin-3-O-arabino furanoside (84) from the $\mathrm{EtOH}$ extract of the whole $M$. diplotricha plant [58].

\subsubsection{M. xanthocentra}

Camargo et al. isolated the flavones isovitexin-2-O- $\alpha$-L-rhamnopyranoside (85), vitexin-2-O- $\alpha$-L-rhamnopyranoside (86), quercetin-3-O-xylopyranoside (81) and quercetin3-O-arabino furanoside (83) from the EtOAc and $\mathrm{BuOH}$ fractions of $M$. xanthocentra aerial parts [121].

\subsubsection{M. hostilis}

Pachter et al. isolated indole alkaloid and $N, N$-dimethyltryptamine (14) from the EtOAc extract of $M$. hostilis roots [122].

\subsubsection{M. artemisiana}

do Nascimento et al. isolated quercetina-3-O-raminosídeo (87), miricetina-3-O-raminoside (88), euphaline,3,5,4-trihydroxy-6,7-dimethoxy flavone (89), flavonolignana (90-93), sitosterol$3-O-\beta$-D glycopyranoside (21), lupeol (22), steroids sitostenone (92), stigmastenone (93), campestenone (94), campesterol (23), stigmasterol (24), sitosterol (25), methyl indole-3carboxilate (95) and indole-3-carboxaldehyde (96) from the $n$-Hex and $\mathrm{MeOH}$ extracts of the leaves and branches of $M$. artemisiana [25].

\subsubsection{M. invisa}

Nana et al. isolated new fatty aldol ester called 17-O-triacontanoylheptadecanal (97) and $\beta$-sitosterol (25), $\alpha$-amyrine (98), lupeol (22), 4-O-methylepinumisoflavone (99), alpinumisoflavone (100), betulinic acid (79), sitosterol 3-O- $\beta$-D-glucopyranoside (21) and epirobinetinidol (101) from the aerial parts of the M. invisa (DCM/MeOH) extracts [28].

\subsubsection{M. scabrella}

Chrestani et al. isolated polysaccharide and sulfated galactomannan (BRS) (102) from the seeds of M. scabrella [123].

\subsubsection{M. somniam}

Gupta et al. isolated the alkaloid tryptamine (103) and N-methyltryptamine (13) from the $\mathrm{MeOH}$ extract of the whole M. somniam plant [124].

\subsubsection{M. pudica}

Whole Plant (Tree) Phytochemicals

Different classes of compounds were isolated from whole $M$. pudica plant (tree). Jose et al. isolated 2-2', $6^{\prime}$-dimethyl-3', $4^{\prime}, 5^{\prime}$-alkyl or hydroxy alkyl substituted phenyl-3-oxy(alkyl or hydoxy alkyl) 5,7-dihydroxy-chromen-4-one (104-107) from the EtOAc fraction of $M$. pudica [50]. One amino acid called L-mimosine (108) was extracted from the hydroalcoholic extract of M. pudica [50] [34,125]. Chukwu et al. isolated triterpenoid glycoside (109) from the crude EtOH extract of the whole M. pudica plant [126]. Tsurumi and Asahi isolated jasmonic acid (110) and abscisic acid (111) from M. pudica [127]. Yuan et al. isolated two new C-glycosyl flavones called 5,7,3', $4^{\prime}$-tetrahydroxy-6-C-[ $\beta$-D-apiose-( $\left.\left.1 \rightarrow 4\right)\right]-\beta$-Dglucopyranosyl flavones (112), 7,8,3', $4^{\prime}$-tetrahy droxyl-6-C-[ $\alpha$-L-rhamnopyranosyl-( $\left.1 \rightarrow 2\right)$ ]$\beta$-D-glucopyranosyl flavones (113), 5,7,4'-trihydroxyl-8-C- $\beta$-D-glucopyranosyl flavones (114), mimosinamine (115), mimosinicacid (116) and tyrosin (117) from the whole M. $p u$ dica plant. Compound (112) is a new compound, and compounds $(\mathbf{1 1 3}, \mathbf{1 1 4})$ were isolated 
for the first time from this plant [128]. Yuan et al. also isolated two new C-glycosyl flavones called 5,7,3', $4^{\prime}$-tetrahydroxy-6-C-[ $\beta$-D-apiose-( $\left.\left.1 \rightarrow 4\right)\right]$ - $\beta$-D-glucopyranosyl flavones (112) and 6,7,3', $4^{\prime}$-tetrahydroxyl-8-C-[ $\alpha$-L-rhamno pyranosyl-( $\left.\left.1 \rightarrow 2\right)\right]-\beta$-D-glucopyranosyl flavone (118) from the M. pudica plant [129].

Aerial Part Phytochemicals

Misra and Tewari isolated six compounds (flavonoids) from the aerial parts of $M$. pudica and identified them as isoquercitrin (119), avicularin (120), apigenin-7-O-D-glucoside (121), cassiaoccidentalin B (122), orientin (123) and isoorientin (124) [130].

Leaf Phytochemicals

Rajalakshmi and Banu reported the presence of chlorophyllin (125) in the $\mathrm{MeOH}$ extract of fresh M. pudica leaves [131]. Josewin et al. isolated the phenolic ketone called 4-(24'-methoxy-24'-methyl-1'-oxo-5'-n-propyl-tetracosanyl)- phenol (126) from the leaves of M. pudica [132]. Kirk et al. isolated three flavonoids called 7, $3^{\prime}, 4^{\prime}$-Triacetoxy-3,8dimethoxyflavone (127), $p$-coumaric acid (128) and 7, $3^{\prime}, 4^{\prime}$-trihydroxy-3,8-dimethoxyflavone (77) from the leaves of M. pudica [133]. Ueda and Yamamura isolated a leaf opening compound called mimopudine (129) and a leaf closing compound called potassium 5-O- $\beta$ D-glucupyranosylgentisate (130) from the leaves of $M$. pudica. This compound mimopudine (129) is responsible for the opening and movements of leaves even at night, while the compound mimopudine (130) is responsible for the closing of the leaves [134,135]. Ueda and Yamamura also isolated different chemical substance such as mimopudine (129), glucupyrano sylgentisate (130), potassium L-malate (131), magnesium potassium trans-aconitate (132) and dimethyl ammonium salt (133) from $M$. pudica leaves. These compounds are responsible for rapid sensitive actions, such as heat and touch, and episodic slow actions, such as nyctinastic actions [136]. Pal et al. isolated tubulin protein (134) (pulvinar callus cells) from the fresh leaves of M. pudica [137]. Khare isolated three compounds as norepinephrine (135), d-pinitol (136) and $\beta$-sitosterol (25) from $M$. pudica leaves [138]. Zhang et al. isolated five flavonoids named as 5,7,3', $4^{\prime}$ tetrahydroxy-6-C-[ $\beta$-D-apiose-( $\left.\left.1 \rightarrow 4\right)\right]-\beta$-Dglycopyranosyl flavone (112), orientin (123), isorientin (124), vitexin (137) and isovitexin (138) from M. pudica leaves [139].

\section{Root Phytochemicals}

Kanga et al. reported a new chroman called 2-hydroxymethyl-chroman-4-one (139) from $M$. pudica roots [140]. Dinda et al. isolated a new sterolglucoside called 4-a,24dimethylcholest-7-en-3 $\beta$-ol-3 $\beta$-D-glucoside (140) along with three other compounds called stigmasterol (24), $\beta$-sitosterol (25) and betulinic acid (79) from the roots of M. pudica [141]. Shu and Ho (2013) isolated two new diterpenoids named 19-O-trans-feruloyl-labd-8(17)en-15,19-diol (141) and 19-O-[(E)-3', $4^{\prime}$-dimethoxy cinnamoyl]-labd-8(17)-en-15,19-diol (142) from the roots of M. pudica [142].

Seed Phytochemicals

Chatterjee and Pakrashi isolated two compounds called D-xylose (143) and D-glucuronic acid 4-O-(3,5-dihydroxybenzoic acid)- $\beta$-D-glucuronide (144) in the form of mucilage from M. pudica seeds [143]. Yadava and Yadav reported a novel compound bufadienolide (hellebrigenin-3-O- $\alpha$-1-rhamnopyranosyl-( $(1 \rightarrow 4)-\mathrm{O}-\beta$-D-galactopyranoside) (145) from the seeds of M. pudica [144].

Stem Phytochemicals

Zaware et al. also isolated nonprotein amino acid called mimosine; [ $\beta$-[N-(3-hydroxy4-oxypyridyl)]- $\alpha$-aminopropionic acid] (108) from the stem of $M$. pudica [145]. 


\section{Pharmacological Activities of Genus Mimosa}

The potential role of the Mimosa genus in traditional medicines encouraged the further biological evaluations of organic extracts and isolated phytoconstituents for potential pharmacological applications. In this section, we summarized the pharmacological activities of genus Mimosa (Table 2).

\subsection{Antiprotozoal Activity}

Antiprotozoals are drugs that are used to treat different infections including babesiosis, microsporidiosis, amebiasis, malaria, leishmaniasis and malaria. These infections are caused by various protozoa. Currently, the treatments of these infections are limited because of toxicity. So, there is a need to find new natural sources to treat these infections with less toxicity. A group of scientists determined the antiprotozoal activity of ten different flavonoids and chromones including 6-methoxy-4-O-metylnaringenin (3), 6-methoxy naringenin (4), santin (5), 4,5,7-trihydroxy-3,6-dimethoxy flavone (6), 6-methoxykaempferol (7), tenuiflorin A (8), tenuiflorin B (9), tenuiflorin C (10), 6-demethoxycapillarisin (11) and 6-demethoxy-4-O-methyl capillarisin (12) isolated from the leaves and flowers of $M$. tenuiflora against $E$. histolytica and $G$. lamblia. The most interesting activity was obtained with (8) $\left(\mathrm{IC}_{50}=41.1 \mu \mathrm{g} / \mathrm{mL}\right)$ against E. histolytica and (5) $\left(\mathrm{IC}_{50}=75.3 \mu \mathrm{g} / \mathrm{mL}\right)$ against $G$. lamblia $[104,105]$.

\subsection{Antimicrobial Activity}

Jain et al. observed the antimicrobial activity of mimonoside A (17), mimonoside B (18), mimonoside C (19), saponin A (51) and saponin B (52) isolated from the leaves and roots of $M$. hamata against different biological strains, such as E. coli, B. subtilis, S. aureus, P. aeruginosa, A. flavus, E. aerogenes, K. pneumoniae, A. niger, C. albicans and $R$. bataticola, using the agar well diffusion method. All compounds showed significant activity against Gram-negative bacteria and fungi, while moderate activity was observed against Gram- positive bacteria compared to standard gentamycin $(10 \mu \mathrm{g} / \mathrm{mL})$ and ketoconazole (100 units $/ \mathrm{mL}$ ). None of the saponins was active against A. niger, A. flavus and C. albicans [119]. Nana et al. measured the antimicrobial activity of new fatty aldol ester called 17-O-triacontanoylheptadecanal (97) and $\beta$-sitosterol (25), $\alpha$-amyrine (98), lupeol (22), 4 O-methylepinumisoflavone (99), alpinumisoflavone (100), betulinic acid (79), sitosterol 3-O- $\beta$-D-glucopyranoside (21) and epirobinetinidol (101) from the aerial parts of $M$. invisa against E. coli, E. aerogenes, S. aureus, P. aeruginosa, K. pneumoniae, S. typhi and C. albicans using the XTT colorimetric assay. Compound (97) displayed antimicrobial activity with MIC values ranging from $64 \mu \mathrm{g} / \mathrm{mL}$ to $256 \mu \mathrm{g} / \mathrm{mL}$. Both compounds showed pronounced activity against $K$. pneumoniae (MIC $=64 \mu \mathrm{g} / \mathrm{mL}$ ). The antimicrobial activity of chlorophyllin (125) extracted from the fresh leaves of M. pudica against P. aeruginosa, E. coli, S. aureus, K. pneomoniae, C. albicans was determined by using the agar well diffusion method [131]. The compound (125) at a concentration of $25 \mu \mathrm{g} / \mathrm{mL}$ showed a significant zone of inhibition against $P$. aeruginosa $=12 \mathrm{~mm}$, E. coli $=8 \mathrm{~mm}$, S. aureus $=14 \mathrm{~mm}, K$. pneumoniae $=13 \mathrm{~mm}$ and C. albicans $=9 \mathrm{~mm}$, while at a concentration of $100 \mu \mathrm{g} / \mathrm{mL}$, P. aeruginosa, E. coli, S. aureus, $K$. pneumoniae and C. albicans $=18 \mathrm{~mm}, 13 \mathrm{~mm}, 19 \mathrm{~mm}$ and $18 \mathrm{~mm}$ inhibition was observed, respectively. The streptomycin sulphate and nystatin (standard) at $10 \mu \mathrm{g} / \mathrm{mL}$ showed a maximum inhibition of $18 \mathrm{~mm}-19 \mathrm{~mm}$ [28]. The antifungal activity of 2-hydroxymethylchroman-4-one (139) isolated from M. pudica roots was checked against various strains, such as P. ultimum, P. capsici, R. solani, B. cinerea, A. panax and S. sclerotiorum, by using the dilution agar plate method. Compound (139) showed a significant $\mathrm{ED}_{50}$ value against $P$. capsici, $S$. sclerotiorum and P. ultimum $=5.7 \mu \mathrm{g} / \mathrm{mL}, 52.1 \mu \mathrm{g} / \mathrm{mL}$ and $54.9 \mu \mathrm{g} / \mathrm{mL}$, respectively [140].

\subsection{Antiviral Activity}

Chrestani et al. measured the antiviral activity of the polysaccharide sulfated galactomannan (BRS) (102) isolated from the seeds of M. scabrella to counter the Herpes simplex virus 1 (HSV-1) at a concentration range of $2 \mu \mathrm{g} / \mathrm{mL}-2.5 \mu \mathrm{g} / \mathrm{mL}$. The (102) exhibited $\mathrm{IC}_{50}$ 
value was less than $2.5 \mu \mathrm{g} / \mathrm{mL}$. Even at a very low concentration $(2.5 \mu \mathrm{g} / \mathrm{mL})$, viral activity was inhibited [123].

\subsection{Antioxidant Activity}

The reactive oxygen species are the cause of many diseases in human beings including cardiovascular and neurological disorders [146]. There is a constant need to counteract the effect of reactive oxidative species to delay the progression on these diseases. Reactive oxygen species and free radicals are scavenged by antioxidants through the termination of a chain reaction, which otherwise can cause damage to cells [147]. Plants are a source of antioxidants and can help to lessen the diseases caused by reactive oxidative species. Natural antioxidants are more potent and less toxic, so there is a need to find more natural sources for well-being. Various species of Mimosa have been screened for antioxidant activity by the application of different assays (Table 2). Jain et al. measured the antioxidant activity of mimonoside A (17), mimonoside B (18), mimonoside C (19), saponin A (51) and saponin B (52) isolated from the leaves and roots of $M$. hamata using the DPPH free radical scavenging assay. Compounds revealed significant $\mathrm{IC}_{50}$ values; (17) $=0.45 \mu \mathrm{g} / \mathrm{mL} ;(\mathbf{1 8})=0.55 \mu \mathrm{g} / \mathrm{mL}$; (19) $=0.60 \mu \mathrm{g} / \mathrm{mL} ;(51)=0.085 \mu \mathrm{g} / \mathrm{mL}$ and $(\mathbf{5 2})=0.10 \mu \mathrm{g} / \mathrm{mL}$, while quercetin (Standard) showed an $\mathrm{IC}_{50}$ value of $0.06 \mu \mathrm{g} / \mathrm{mL}$ [119]. The amino acid $L$-mimosine (108) was extracted from the hydroalcoholic extract of M. pudica, and the antioxidant activity was determined by the DPPH radical scavenging assay. The compound (108) at a concentration of $250 \mu \mathrm{M}$ showed significant activity $\left(\mathrm{IC}_{50}=233.06 \mu \mathrm{M}\right)[34,125]$.

\subsection{Antiproliferative Activity}

Chiou et al. investigated the antiproliferative activity of isolated compounds called diplomero terpenoids A-F (54-59), two new chalcone-lignoids called diplochalcolins A and B $(\mathbf{6 0}, 61)$ and 13 known compounds called hydnocarpin (62), 7,4-dihydroxyflavone (63), chrysoeriol (64), apigenin (65), diplotrin B (66), 2-hydroxy-3,7, $4^{\prime}, 8,5^{\prime}$-pentamethoxyflavone (67), hernancorizin (68), diplotasin D (69), 7-hydroxy-8-methoxychromone (70), (+)-syringaresinol (71), 4-hydroxy3,5-dimethoxybenzoic acid (72), $\beta$-sitosterol (25) and $\beta$-sitosterol glucoside (73) isolated from $M$. diplotricha roots against human hepatoblastoma $\mathrm{HepG}_{2}$ cells by the Sulforhodamine $\mathrm{B}$ assay (SRB). Compound 54 displayed growth inhibition activity with $\mathrm{GI}_{50}=8.6 \mu \mathrm{M}$, although the $\mathrm{GI}_{50}$ values of the compounds (55-60) were greater than $10 \mathrm{Mm}$ [120].

\subsection{Cytotoxic Activity}

Cancer is an uncontrolled and abnormal symmetric growth of body cells [148]. Different cancers were observed in humans, such as in the liver, stomach, lung, breast, prostate, thyroid and cervix. Bioactive compounds isolated from plants are used in curing cancer because they are inexpensive, nontoxic and easily available as compared to synthetic compounds. The bioactive compounds in plants induced apoptosis in infective cancer cells and also helped to restore chemotherapy sensitivity [149]. Lin et al. reported the cytotoxic activity of four 5-deoxyflavones called diplotrin A (74), diplotrin B (66), diplotrin C (75), diplotasin D (69), 5-methoxyhydnocarpin-D (76), 7,3' $4^{\prime}$-trihydroxy-3,8dimethoxyflavone (77), 2-hydroxy-3,7,8,4,5 pentamethoxy flavone (67), hernancorizin (68), $5,3^{\prime}$-di-O-methylluteolin (78), betulinic acid (79), luteolin (80), quercetin (38), quercetin-3-Oxylopyranoside (81), myricetin-3-O-xylopyranoside (82), quercetin-3-O-arabino furanoside (83) and myricetin-3-O-arabino furanoside (84) isolated from the whole $M$. diplotricha plant against the A549, HT-29, AGS and $\mathrm{PC}_{3}$ human cancer cell lines using the SRB assay. Compounds (66) and (76) presented the powerful antiproliferative activity with $\mathrm{GI}_{50}$ values of $(66)=2.7 \mu \mathrm{M}, 1.7 \mu \mathrm{M}, 7.5 \mu \mathrm{M}$, and $20.8 \mu \mathrm{M}$, respectively and (76) $20.3 \mu \mathrm{M}, 24.8 \mu \mathrm{M}$, $4.1 \mu \mathrm{M}$, and $2.3 \mu \mathrm{M}$, respectively toward the four human cancer cell lines, while all the other compounds were found $>10 \mathrm{Mm}$ [58]. Chrestani et al. measured the cytotoxic potential of sulfated galactomannan (BRS) (102) isolated from the seeds of M. scabrell against the Vero and MA-104 human cell lines by the MTT assay. At a concentration of $\geq 39 \mu \mathrm{g} / \mathrm{mL}$, BRS reduced by $15 \%$ the viability of Vero cells $\left(\mathrm{CC}_{50}=454 \mu \mathrm{g} / \mathrm{mL}\right)$, while 
at a concentration of $625 \mu \mathrm{g} / \mathrm{mL}$, BRS reduced by $24 \%$ the viability of MA-104 [123]. Scientists screened the cytotoxicity of the isolated $\left(2-\left(2^{\prime}, 6^{\prime}\right.\right.$-dimethyl- $3^{\prime}, 4^{\prime}, 5^{\prime}$-alkyl or hydroxy alkyl substituted phenyl)-3-oxy-(alkyl or hydoxy alkyl) 5,7-dihydroxy-chromen-4-one) (104-107) from M. pudica using the MTT assay against human lung adenocarcinoma (A549) and the erythroleukemic cell line (K562). The compound showed significant $\mathrm{IC}_{50}$ against A549 $=76.67 \mu \mathrm{g} / \mathrm{mL}$ and $\mathrm{K} 562=287.63 \mu \mathrm{g} / \mathrm{mL}$, while the positive control Doxorubicin showed an $\mathrm{IC}_{50}$ value of $2.76 \mu \mathrm{g} / \mathrm{mL}$ and $\mathrm{K} 562=4.72 \mu \mathrm{g} / \mathrm{mL}$ against A549 [50]. The cytotoxic potential of the amino acid L-Mimosine (108) extracted from M. pudica by the MTT assay against the daudi cell line was reported. After $72 \mathrm{~h}$, the compound (108) showed an $\mathrm{IC}_{50}$ value of $86.61 \mu \mathrm{M}$. Compound (108) act as powerful inhibitors of cell proliferation and showed remarkable cytotoxic activity $[34,125]$.

\section{Marker Compounds of Genus Mimosa}

Some of the identified compounds from genus Mimosa are specific marker components of this genus. Two chalcones called kukulkan A (1) and kukulkan B (2) [103] and different terpenoidal saponins called mimonoside A (17), mimonoside B (18) and mimonoside C (19) $[13,108,109]$ are specifically identified in M. tenuiflora. Six new meroterpenoids called diplomero terpenoids A-F (54-59), two new chalcone-lignoids called diplochalcolins A and B $(60,61)$ have been identified in M. diplotricha roots [120]. Similarly, four 5-deoxyflavones called diplotrin A (74), diplotrin B (66), diplotrin C (75) and diplotasin D (69) were specifically found in the whole $M$. diplotricha plant [58]. One amino acid called L-mimosine (108) was extracted from the hydroalcoholic extract of M. pudica [50]. Novel C-glycosyl flavones called 5,7,3' $4^{\prime}$-tetrahydroxy-6-C-[ $\beta$-D-apiose-( $\left.\left.1 \rightarrow 4\right)\right]-\beta$-D-glucopyranosyl flavones (112), $7,8,3^{\prime}, 4^{\prime}$-tetrahy droxyl-6-C-[ $\alpha$-L-rhamnopyranosyl-( $\left.\left.1 \rightarrow 2\right)\right]-\beta$-D-glucopyranosyl flavones (113), 5,7,4'-trihydroxyl-8-C- $\beta$-D-glucopyranosyl flavones (114), mimosinamine (115) and mosinicacid (116) were isolated from the whole M. pudica plant [128]. A leaf opening compound called mimopudine (129) and a leaf closing compound called potassium 5-O$\beta$-D-glucupyranosylgentisate (130) were isolated from the leaves of $M$. pudica. This compound called mimopudine (129) is responsible for the opening and movements of leaves even at night, while the compound mimopudine (130) is responsible for the closing of leaves [134,135]. Similarly, potassium L-malate (131), magnesium potassium trans-aconitate (132) and dimethylammonium salt (133) from M. pudica leaves were isolated, and these compounds were responsible for rapid sensitive actions, such as heat and touch, and episodic slow actions, such as nyctinastic actions [136]. A new chroman called 2-hydroxymethylchroman-4-one (139) [140] and a new sterolglucoside 4-a,24-dimethylcholest-7-en-3 $\beta$-ol-3 $\beta$ D-glucoside (140) were specifically identified from the roots of $M$. pudica [141]. Two new diterpenoids named 19-O-trans-feruloyl-labd-8(17)-en-15,19-diol (141) and 19-O-[(E)-3', $4^{\prime}$ dimethoxy cinnamoyl]-labd-8(17)-en-15,19-diol (142) were isolated from the roots of $M$. pudica [142]. A novel compound called bufadienolide (hellebrigenin-3-O- $\alpha$-l-rhamnopyranosyl$(1 \rightarrow 4)$-O- $\beta$-D-galactopyranoside) (145) was specifically isolated from the seeds of $M$. $p u$ dica [144].

\section{Conclusions and Future Perspectives}

This review summarized the isolated phytochemical and pharmacological characteristics of the Mimosa genus. Out of 400 species only 25 have been chemically studied, while compounds belonging to different chemical classes have been isolated in the Mimosa species, such as alkaloids, chalcones, flavonoids, indoles, terpenes, terpenoids, saponins, steroids, amino acids, glycosides, flavanols, phenols, lignoids, polysaccharides, lignins and fatty esters. Significant bioactivities, such as antimicrobial, cytotoxic, antioxidant, antiprotozoal, antiviral and antiproliferative, were discussed in this review. In this review, M. pudica was the most studied specie. This review also covered the qualitative and quantitative analysis of phytochemicals, such as flavonoids, steroids, saponins, alkaloids, coumarins, tannins and terpenoids, in the genus Mimosa. This review focused on the medicinal aspects of the Mimosa species and may provide a comprehensive understanding of the prospective of this 
genus as a foundation of medicine, supplement and nourishment. Several studies were performed to establish the pharmacological potential by identifying the bioactive secondary metabolites associated with the respective activities. Many studies have been carried out that show this genus possesses huge potential for new drug sources, but there are still gaps, which are noteworthy. Few species of this genus have been explored, so there is a need to explore all species of this genus to find their potential medicinal values for well-being. Secondly, there is a need to provide detailed mechanistic studies on the pharmacology to provide a good understanding of the application of the Mimosa species as a source of potential medicines. Thirdly, further studies are required to explore safety aspects of the diverse range of the Mimosa species, including chronic toxicity with a determination of the molecular pathways of the health-promoting features of this genus, and attempts are required to isolate more bioactive compounds of this genus.

Author Contributions: Conceptualization, I.M. and K.R.; methodology, T.R.; software, I.M., S.I. and K.R.; validation, M.B.; investigation, M.B. and T.R.; resources, K.R. and A.S.; data curation, I.M. and K.R.; writing—original draft preparation, I.M. and K.R.; writing—review and editing, M.B., K.R. and A.S.; visualization, K.R.; supervision, K.R. All authors have read and agreed to the published version of the manuscript.

Funding: The APC was funded by TU Delft, The Netherlands.

Institutional Review Board Statement: Not applicable.

Informed Consent Statement: Not applicable.

Data Availability Statement: The data presented in this study are available on request from the corresponding authors.

Conflicts of Interest: All the authors declare no conflict of interest.

\section{Abbreviations}

Butanol $=\mathrm{BuOH}$, Hexane $=$ Hex, Acetone $=\mathrm{ACE}$, Methanol $=\mathrm{MeOH}$, Ethylacetate $=$ EtOAc, Ethanolic $=\mathrm{EtOH}$, Diethyl ether $=\mathrm{DEE}, \mathrm{Hydroalcohol}=\mathrm{HyOH}$, Aqueous $=$ Aq., Pet. ether $=\mathrm{PE}$, Chloroform $=\mathrm{CF}$, Dicholomethane $=\mathrm{DCM}$, Acetic acid $=$ AA.

\section{References}

1. Ahuchaogu, A.A.; Chukwu, O.J.; Echeme, J.O. Secondary Metabolites from Mimosa Pudica: Isolation, Purification and NMR Characterization. IOSR J. Appl. Chem. 2017, 10, 15-20. [CrossRef]

2. Silva, A.S.; Araújo, S.B.; Souza, D.C.; Silva, F.A. Study of the $\mathrm{Cu}, \mathrm{Mn}, \mathrm{Pb}$ and $\mathrm{Zn}$ dynamics in soil, plants and bee pollen from the region of Teresina (PI), Brazil. An. Acad. Bras. Ciências 2012, 84, 881-889. [CrossRef] [PubMed]

3. Muddiman, S.; Hodkinson, I.; Hollis, D. Legume-feeding psyllids of the genus Heteropsylla (Homoptera: Psylloidea). Bull. Entomol. Res. 1992, 82, 73-117. [CrossRef]

4. Amalraj, T.; Ignacimuthu, S. Hyperglycemic effect of leaves of Mimosa pudica Linn. Fitoterapia 2002, 73, 351-352. [CrossRef]

5. Camargo-Ricalde, S.L. Descripción, distribución, anatomía, composición química y usos de Mimosa tenuiflora (FabaceaeMimosoideae) en México. Rev. Biol. Trop. 2000, 48, 939-954. [PubMed]

6. Galinato, M.I.; Moody, K.; Piggin, C.M. Upland Rice Weeds of South and Southeast Asia; IRRI: Los Baños, Philippine, $1999 ;$ p. 161.

7. Hussain, N.; Modan, M.H.; Shabbir, S.G.; Zaidi, S. Antimicrobial principles in Mimosa hamata. J. Nat. Prod. 1979, 42, 525-527. [CrossRef] [PubMed]

8. Mahmood, A.; Mahmood, A.; Qureshi, R.A. Antimicrobial activities of three species of family mimosaceae. Pak. J. Pharm. Sci. 2012, 25, 203-206.

9. Joyamma, V.; Rao, S.; Hrishikeshavan, H.; Aroor, A.; Kulkarni, D. Biochemical mechanisms and effects of Mimosa pudica (Linn) on experimental urolithiasis in rats. Indian J. Exp. Biol. 1990, 28, 237-240.

10. Gupta, R.; Vairale, M.; Deshmukh, R.; Chaudhary, P.; Wate, S.R. Ethnomedicinal uses of some plants used by Gond tribe of Bhandara district, Maharashtra. Indian J. Tradit. Knowl. 2010, 9, 713-717.

11. Lemes, P.G.; Castro, A.; Zanuncio, J. Oncideres ocularis (Coleoptera: Cerambycidae) Girdling Mimosa bimucronata (Fabaceae) in Brazil. Fla. Entomol. 2014, 97, 1240-1243. [CrossRef]

12. Lorenzi, H. Árvores Brasileiras: Manual de Identificação e Cultivo de Plantas Arbóreas Nativas do Brasil, 2nd ed.; Editora Plantarum: Nova Odessa, SP, Brazil, 1998; p. 10. 
13. Jiang, Y.; Massiot, G.; Lavaud, C.; Teulon, J.-M.; Guéchot, C.; Haag-Berrurier, M.; Anton, R. Triterpenoid glycosides from the bark of Mimosa tenuiflora. Phytochemistry 1991, 30, 2357-2360. [CrossRef]

14. Ueda, M.; Takada, N.; Yamamura, S. Molecular approach to the nyctinastic movement of the plant controlled by a biological clock. Int. J. Mol. Sci. 2001, 2, 156-164. [CrossRef]

15. Fernandes, G.; Ferrari, R.R. Fruits of Mimosa foliolosa (Fabales: Fabaceae) as Sleeping Shelter for Megachile (Pseudocentron) botucatuna (Hymenoptera: Megachilidae). Neotrop. Entomol. 2012, 41, 518-520. [CrossRef]

16. Baggio, A.J.; Carpanezzi, A.A. Exploração Seletiva do Sub-Bosque: Uma Alternativa para Aumentar a Rentabilidade dos Bracatingais; Embrapa Florestas-Circular Técnica (INFOTECA-E); Empresa Brasileira de Pesquisa Agropecuária: Brasilia, Brazil, 1998.

17. Simon, M.F.; Grether, R.; de Queiroz, L.P.; Särkinen, T.E.; Dutra, V.F.; Hughes, C.E. The evolutionary history of Mimosa (Leguminosae): Toward a phylogeny of the sensitive plants. Am. J. Bot. 2011, 98, 1201-1221. [CrossRef] [PubMed]

18. Nworgu, F.; Egbunike, G. Nutritional Potential of Centrosema pubescens Mimosa invisa and Pueraria phaseoloides Leaf Meals on Growth Performance Responses of Broiler Chickens. Am. J. Exp. Agric. 2013, 3, 506. [CrossRef]

19. Erkan, G.; Şengül, K.; Kaya, S. Dyeing of white and indigo dyed cotton fabrics with Mimosa tenuiflora extract. J. Saudi Chem. Soc. 2014, 18, 139-148. [CrossRef]

20. Silva, T.M.S.; dos Santos, F.P.; Evangelista-Rodrigues, A.; da Silva, E.M.S.; da Silva, G.S.; de Novais, J.S.; dos Santos, F.d.A.R.; Camara, C.A. Phenolic compounds, melissopalynological, physicochemical analysis and antioxidant activity of jandaíra (Melipona subnitida) honey. J. Food Compos. Anal. 2013, 29, 10-18. [CrossRef]

21. Vasconcelos do Nascimento, C.; Albuquerque Pontes Filho, R.; Artur, A.; Costa, M. Application of poultry processing industry waste: A strategy for vegetation growth in degraded soil. Waste Manag. 2014, 36, 316-322. [CrossRef]

22. Apolinário, V.; Dubeux, J., Jr.; Lira, M.; Luiz, R.; Mello, A.; Santos, M.; Sampaio, E.V.S.B.; Muir, J. Tree Legumes Provide Marketable Wood and Add Nitrogen in Warm-Climate Silvopasture Systems. Agron. J. 2015, 107, 1915-1921. [CrossRef]

23. Chiocchio, I.; Mandrone, M.; Sanna, C.; Maxia, A.; Tacchini, M.; Poli, F. Screening of a hundred plant extracts as tyrosinase and elastase inhibitors, two enzymatic targets of cosmetic interest. Ind. Crops Prod. 2018, 122, 498-505. [CrossRef]

24. Durgadevi, G.; Karthika, N. Screening of phytochemicals and pharmacological studies on Mimosa pudica L. Asian J. Innov. Res. 2018, 3, 19-28.

25. do Nascimento, I.A.; Braz-Filho, R.; de Carvalho, M.G.; Mathias, L.; de Alcântara Fonseca, F. Flavonoides e Outros Compostos Isolados de Mimosa artemisiana Heringer e Paula. Quim. Nova 2012, 35, 2159-2164. [CrossRef]

26. Mathew, J.; Joy, J.K.; Vazhacharickal, P.; Sajeshkumar, N.K. Phytochemical Analysis And Invitro Hemostatic Activity Of Mimosa Pudica, Hemigraphis Colorata And Chromolaena Odorata Leaf Extracts. CIBTech J. Pharm. Sci. 2016, 5, 16-34.

27. Oliveira, F.F.M.; Barbosa, K.M.K.M.; de Oliveira, G.F.; Dantas, I.M.; Camacho, R.G.V. Micropropagação de Mimosa caesalpiniaefolia Benth. a partir de segmentos nodais e ápices caulinares. Rev. Caatinga 2007, 20, 152-159.

28. Nana, F.; Sandjo, L.P.; Keumedjio, F.; Kuete, V.; Ngadjui, B.T. A new fatty aldol ester from the aerial part of Mimosa invisa (Mimosaceae). Nat. Prod. Res. 2012, 26, 1831-1836. [CrossRef]

29. Tunna, T.; Zaidul, I.; Ahmed, Q.; Ghafoor, K.; Al-Juhaimi, F.; Uddin, M.; Hasan, M.; Ferdous, S. Analyses and profiling of extract and fractions of neglected weed Mimosa pudica Linn. traditionally used in Southeast Asia to treat diabetes. S. Afr. J. Bot. 2015, 99, 144-152. [CrossRef]

30. Sutar, N.; Sutar, U.N.; Behera, B.C. Antidiabetic activity of the leaves of Mimosa pudica Linn. in albino rats. J. Herb. Med. Toxicol. 2009, 3, 123-126.

31. Saraswat, R.; Pokharkar, R. GCMS studies of mimosa pudica. Int. J. PharmTech Res. 2012, 4, $93-98$.

32. Saxena, R.; Sharma, R.; Chandra Nandy, B.; Hardainiyan, S. The Study Of Phenolic Compounds And Antioxidant Potential Of Crude Extract And Fractions Of Mimosa Hamata. Int. Res. J. Pharm. 2017, 8, 83-86. [CrossRef]

33. Almalki, M. In-Vitro Antioxidant Properties of the Leaf Extract of Mimosa pudica Linn. Indian J. Sci. Technol. 2016, 9, 1-6. [CrossRef]

34. Parmar, F.; Kushawaha, N.; Highland, H.; George, L.-B. In vitro antioxidant and anticancer activity of Mimosa pudica linn extract and L-Mimosine on lymphoma daudi cells. Cancer Cell 2015, 1, 100-104.

35. Silva, M.J.D.; Vilegas, W.; da Silva, M.A.; de Moura, C.F.G.; Ribeiro, F.A.P.; da Silva, V.H.P.; Ribeiro, D.A. Mimosa (Mimosa caesalpiniifolia) prevents oxidative DNA damage induced by cadmium exposure in Wistar rats. Toxicol. Mech. Methods 2014, 24, 567-574. [CrossRef] [PubMed]

36. Chandarana, M.; Singh, R.; Jasrai, Y. Determination of the phenolic and flavonoid contents in Mimosa hamataWilld., as well as their radical scavenging activity. Int. J. Pharmacogn. Phitochem. 2013, 28, 1121-1126.

37. Borneo, R.; León, A.; Aguirre, A.; Ribotta, P.; Cantero, J. Antioxidant capacity of medicinal plants from the Province of Cordoba (Argentina) and their in vitro testing in model food system. Food Chem. 2009, 112, 664-670. [CrossRef]

38. Prathima, C.; Shashikumara, T.T.; Jayanthi, M.K. Evaluation of anticonvulsant activity of Mimosa pudica root linn in swiss albino mice. Int. J. Pharm. Pharm. Sci. 2016, 8, 49-52.

39. Jiang, Y.; You, X.-Y.; Fu, K.-L.; Yin, W.-L. Effects of Extract from Mangifera indica Leaf on Monosodium Urate Crystal-Induced Gouty Arthritis in Rats. Evid. Based Complement. Altern. Med. 2012, 2012, 967573. [CrossRef]

40. Sampaio Octaviano de Souza, R.; Albuquerque, U.; Monteiro, J.; Lúcia Cavalcanti de Amorim, E. Jurema-Preta (Mimosa tenuiflora [Willd.] Poir.): A Review of its Traditional Use, Phytochemistry and Pharmacology. Braz. Arch. Biol. Technol. 2008, 51, 937-947. [CrossRef] 
41. Patel, N.K.; Bairwa, K.; Gangwal, R.; Jaiswal, G.; Jachak, S.M.; Sangamwar, A.T.; Bhutani, K.K. 2'-Hydroxy flavanone derivatives as an inhibitors of pro-inflammatory mediators: Experimental and molecular docking studies. Bioorgan. Med. Chem. Lett. 2015, 25, 1952-1955. [CrossRef] [PubMed]

42. Borsato, D.M.; Prudente, A.S.; Döll-Boscardin, P.M.; Borsato, A.V.; Luz, C.F.; Maia, B.H.; Cabrini, D.A.; Otuki, M.F.; Miguel, M.D.; Farago, P.V. Topical anti-inflammatory activity of a monofloral honey of Mimosa scabrella provided by Melipona marginata during winter in Southern Brazil. J. Med. Food 2014, 17, 817-825. [CrossRef] [PubMed]

43. Bitencourt, M.; Conceição Jerônimo de Souza Lima, M.; Torres-Rêgo, M.; Fernandes, J.; Silva-Júnior, A.; Tambourgi, D.; Maria Zucolotto, S.; Fernandes-Pedrosa, M. Neutralizing Effects of Mimosa tenuiflora Extracts against Inflammation Caused by Tityus serrulatus Scorpion Venom. BioMed Res. Int. 2014, 2014, 378235. [CrossRef] [PubMed]

44. Cruz, M.P.; Andrade, C.M.F.; Silva, K.O.; de Souza, E.P.; Yatsuda, R.; Marques, L.M.; David, J.P.; David, J.M.; Napimoga, M.H.; Clemente-Napimoga, J.T. Antinoceptive and Anti-inflammatory Activities of the Ethanolic Extract, Fractions and Flavones Isolated from Mimosa tenuiflora (Willd.) Poir (Leguminosae). PLoS ONE 2016, 11, e0150839. [CrossRef]

45. Kumar Vikram, P.; Malvi, R.; Jain, D. evaluation of analgesic and anti-inflammatory potential of mimosa pudica linn. Int. J. Curr. Pharm. Res. 2015, 4, 47-50.

46. Ganguly, M.; Devi, N.; Mahanta, R.; Borthakur, M.K. Effect of Mimosa pudica root extract on vaginal estrous and serum hormones for screening of antifetility activity in albino mice. Contraception 2008, 76, 482-485. [CrossRef]

47. Le Tran, Q.; Tezuka, Y.; Ueda, J.-Y.; Nhan, N.; Maruyama, Y.; Begum, K.; Kim, H.; Wataya, Y.; Kim Tran, Q.; Kadota, S. In Vitro Antiplasmodial Activity of Antimalarial Medicinal Plants Used in Vietnamese Traditional Medicine. J. Ethnopharmacol. 2003, 86, 249-252. [CrossRef]

48. Marimuthu, S.; Rahuman, A.; Rajakumar, G.; Thirunavukkarasu, S.; Kirthi, V.; Chidambaram, J.; Bagavan, A.; Abduz Zahir, A.; Elango, G.; Kamaraj, D.C. Evaluation of green synthesized silver nanoparticles against parasites. Parasitol. Res. 2010, 108, 1541-1549. [CrossRef] [PubMed]

49. Paul, J.; Khan, S.; Mohammed, S.; Asdaq, S. Wound healing evaluation of chloroform and methanolic extracts of mimosa pudica roots in rats. Int. J. Biol. Med. Res. 2010, 1, 223-227.

50. Jose, J.; Dhanya, A.; Haridas, K.R.; Kumar, T.S.; Jayaraman, S.; Variyar, E.J.; Sudhakaran, S. Structural characterization of a novel derivative of myricetin from Mimosa pudica as an anti-proliferative agent for the treatment of cancer. Biomed. Pharmacother. 2016, 84, 1067-1077. [CrossRef]

51. Monção, B.N.; Araújo, Q.B.; Silva, D.J.; Lima, J.D.; Ferreira, M.P.; Airoldi, P.F.; Pessoa, C.; Citó, M.A. Assessing Chemical Constituents of Mimosa caesalpiniifolia Stem Bark: Possible Bioactive Components Accountable for the Cytotoxic Effect of M. caesalpiniifolia on Human Tumour Cell Lines. Molecules 2015, 20, 4204-4224. [CrossRef]

52. Molina, M.; Contreras, C.; Tellez-Alcantara, P. Mimosa pudica may possess antidepressant actions in the rat. Phytomedicine 1999, 6, 319-323. [CrossRef]

53. Saifuddin Khalid, M.; Shah, J.; Suresh, D.K.; Singh, R.; Narasimha Reddy, I.V.; Kumar, S. Evaluation of anti-diarrhoeal potential of ethanolic extract of Mimosa pudica leaves. Int. J. Green Pharm. 2011, 5, 75. [CrossRef]

54. Balakrishnan, N.; Bhaskar, V.; Jayakar, B.; Sangameswaran, B. Antibacterial activity of Mimosa pudica, Aegle marmelos and Sida cordifolia. Pharmacogn. Mag. 2006, 2, 198-199.

55. Sowmya, A.; Ananthi, T. Hypolipidemic activity of Mimosa pudica Linn on butter induced hyperlipidemia in rats. Asian J. Res. Pharm. Sci. 2011, 1, 123-126.

56. Purkayastha, A.; Chakravarty, P.; Dewan, B. hypolipidemic effect of ethanolic extract of mimosa pudica leaves on dyslipidemia following hepatic injury induced by carbon tetrachloride in albino rats. Int. J. Pharm. Sci. Res. 2016, 7, 3284-3290.

57. Kumaresan, R.; Veerakumar, S.; Elango, V. A study on hepatoprotective activity of Mimosa pudica in albino rats. Int. J. Pharm. Phytochem. Res. 2015, 7, 337-339.

58. Lin, L.-C.; Chiou, C.-T.; Cheng, J.-J. 5-Deoxyflavones with cytotoxic activity from Mimosa diplotricha. J. Nat. Prod. 2011, 74, 2001-2004. [CrossRef]

59. Jain, S.; Jain, R.; Vlietinck, A. In vivo and in vitro antimicrobial efficacy of Mimosa hamata. Indian J. Biotechnol. 2004, 3, $271-273$.

60. Mehta, B.K.; Savita Sharma, K.; Dubey, A. 4-Ethylgallic acid from two Mimosa species. Phytochemistry 1988, 27, 3004-3005. [CrossRef]

61. Mehmood, N.; Zubair, M.; Rızwan, K.; Rasool, N.; Shahid, M.; Ahmad, V. Antioxidant, antimicrobial and phytochemical analysis of Cichorium intybus seeds extract and various organic fractions. Iran. J. Pharm. Res. 2012, 11, 1145-1151.

62. Aslam, F.; Riaz, M.; Zubair, M.; Rizwan, K.; Abbas, M.; Bukhari, T.H.; Bukhari, I.H. Antioxidant, haemolytic activities and GC-MS profiling of Carissa carandas roots. Int. J. Phytomed. 2012, 3, 567-578.

63. Bari, M.N.; Zubair, M.; Rizwan, K.; Rasool, N.; Bukhari, I.H.; Akram, S.; Bokhari, T.H.; Shahid, M.; Hameed, M.; Ahmad, V.U. Biological activities of Opuntia Monacantha cladodes. J. Chem. Soc. Pak. 2012, 34, 990-995.

64. Imran, M.; Rasool, N.; Rizwan, K.; Zubair, M.; Riaz, M.; Zia-Ul-Haq, M.; Rana, U.A.; Nafady, A.; Jaafar, H.Z. Chemical composition and Biological studies of Ficus benjamina. Chem. Cent. J. 2014, 8, 12. [CrossRef] [PubMed]

65. Rizwan, K.; Zubair, M.; Rasool, N.; Mahmood, A.; Ercisli, S.; Tareen, R.B.; Zia-Ul-Haq, M. Compositional studies and antioxidant potential of Fruit of Zizyphus oxyphylla EDGEW. Oxid. Commun. 2016, 39, 1309-1322.

66. Ashraf, S.N.; Zubair, M.; Rizwan, K.; Tareen, R.B.; Rasool, N.; Zia-Ul-Haq, M.; Ercisli, S. Compositional studies and Biological activities of Perovskia abrotanoides Kar. oils. Biol. Res. 2014, 47, 12. [CrossRef] 
67. Rasool, N.; Tehseen, H.; Riaz, M.; Rizwan, K.; Zubair, M.; Mahmood, Y.; Iqbal, M.; Bukhari, I.H. Cytotoxicity studies and antioxidant potential of Acacia nilotica roots. Int. J. Chem. Biochem. Sci. 2013, 3, 34-41.

68. Rasool, N.; Afzal, S.; Riaz, M.; Rashid, U.; Rizwan, K.; Zubair, M.; Ali, S.; Shahid, M. Evaluation of antioxidant activity, cytotoxic studies and GC-MS profiling of matthiola incana (stock flower). Legume Res. Int. J. 2013, 36, 21-32.

69. Khan, S.A.; Rasool, N.; Riaz, M.; Nadeem, R.; Rashid, U.; Rizwan, K.; Zubair, M.; Bukhari, I.H.; Gulzar, T. Evaluation of Antioxidant and Cytotoxicity Studies of Clerodendrum inerme. Asian J. Chem. 2013, 25, 7457-7462. [CrossRef]

70. Zubair, M.; Rizwan, K.; Rashid, U.; Saeed, R.; Saeed, A.A.; Rasool, N.; Riaz, M. GC/MS profiling, in vitro antioxidant, antimicrobial and haemolytic activities of Smilax macrophylla leaves. Arab. J. Chem. 2017, 9, S1435-S1442. [CrossRef]

71. Zia-Ul-Haq, M.; Stanković, M.S.; Rizwan, K.; Feo, V.D. Grewia asiatica L., a food plant with multiple uses. Molecules 2013, 18, 2663-2682. [CrossRef]

72. Zubair, M.; Bibi, Z.; Rizwan, K.; Rasool, N.; Zahoor, A.F.; Riaz, M. In Vitro Antimicrobial and Haemolytic Studies of Bambusa arundinaceae leaves. J. Appl. Pharm. Sci. 2013, 3, 111-115.

73. Riaz, M.; Rasool, N.; Bukhari, I.H.; Shahid, M.; Zubair, M.; Rizwan, K.; Rashid, U. In vitro antimicrobial, antioxidant, cytotoxicity and GC-MS analysis of Mazus goodenifolius. Molecules 2012, 17, 14275-14287. [CrossRef]

74. Rizwan, K.; Zubair, M.; Rasool, N.; Riaz, M.; Zia-Ul-Haq, M.; de Feo, V. Phytochemical and biological studies of Agave attenuata. Int. J. Mol. Sci. 2012, 13, 6440-6451. [CrossRef] [PubMed]

75. Riaz, M.; Rasool, N.; Bukhari, I.; Rizwan, K.; Zubair, M.; Javed, F.; Altaf, A.; Qayyum, H. Antioxidant, Antimicrobial Activity and GC-MS analysis of Russelia equsetiformis Essential Oils. Oxid. Commun. 2012, 35, 1027-1037.

76. Sharma, P.V.; Ahmad, Z.A. Present status of research on genus: Mimosa. Anc. Sci. Life 1987, 7, $21-29$.

77. Singh, R.; Jasrai, Y.T. Mimosa hamata (Willd.), a plant with antipathogenic properties. Int. J. Med. Aromat. Plants $2012,2,677-682$.

78. Jasuja, N.D.; Saxena, R.; Chandra, S.; Bhargava, S.; Joshi, S. Pharmacological evaluation of an ethnomedicinal and endangered desert plant: Mimosa hamata. J. Biol. Sci. 2014, 14, 52-59. [CrossRef]

79. Muhammad, G.; Hussain, M.A.; Jantan, I.; Bukhari, S.N.A. Mimosa pudica L., a high-value medicinal plant as a source of bioactives for pharmaceuticals. Compr. Rev. Food Sci. Food Saf. 2016, 15, 303-315. [CrossRef] [PubMed]

80. Sanaye, M.; Joglekar, C.; Pagare, N. Mimosa-A brief overview. J. Pharmacogn. Phytochem. 2015, 4, 182-187.

81. Majeed, I.; Rizwan, K.; Ashar, A.; Rasheed, T.; Amarowicz, R.; Kausar, H.; Zia-Ul-Haq, M.; Marceanu, L.G. A Comprehensive Review of the Ethnotraditional Uses and Biological and Pharmacological Potential of the Genus Mimosa. Int. J. Mol. Sci. 2021, 22, 7463. [CrossRef]

82. Haddad, H.; Seyed Hoseini, E.; Nikzad, H.; Hossein Aarabi, M. Pharmacological properties of medicinal herbs by focus on secondary metabolites. Life Sci. J. 2012, 9, 509-520.

83. Oliveira, L.M.B.; Macedo, I.T.F.; Vieira, L.S.; Camurça-Vasconcelos, A.L.F.; Tomé, A.R.; Sampaio, R.A.; Louvandini, H.; Bevilaqua, C.M.L. Effects of Mimosa tenuiflora on larval establishment of Haemonchus contortus in sheep. Vet. Parasitol. 2013, 196, 341-346. [CrossRef]

84. Racadio, S.P. The Medicinal Prospects of Makahiya (Mimosa Pudica Linn) Plant. Adv. Life Sci. 2016, 6, 7-12.

85. Ahuchaogu, A.; Chukwu, J.; Obike, A.; Ugonna Oha, T.; Bull, J.; Echeme, O. Quantitative Determination of Secondary Metabolites and Antibacterial Activity of Mimosa Pudica. Int. J. Med. Plants Nat. Prod. 2017, 3, 2454-7999.

86. Sheeba, D.G.; Gomathi, K.S.; Citarasu, D. Anti-Mycobacterial and Phytochemical Investigation of Methanol Extracts of Few Medicinal Plants. J. Chem. Pharm. Sci. 2015, 8, 480-486.

87. Mahadevan, M.V.; Ramaswamy, R.S.; Banumathi, V. Mimosa pudica exerts neuroprotection against mpp+ induced neurotoxicity in shsy5y cell lines-an in vitro model of anti-parkinsonism. Int. J. Pharm. Pharm. Sci. 2016, 9, 21.

88. Ramesh, S.; Karthikeyan, K.; Chandran, C. Photochemical screening and pharmacognostic studies on Mimosa pudica L. (Sensitive plant). Int. J. Fauna Biol. Stud. 2017, 4, 170-175.

89. Chinnathambi, A.; Sathasivam, A. Analysis of the phytochemical constituents of Mimosa pudica and determination of their antimicrobial activity. In Proceedings of the Biotech Congress, Beijing, China, 13-15 July 2015; p. 61.

90. Harborne, J.B.; Williams, C.A. Anthocyanins and other flavonoids. Nat. Prod. Rep. 2001, 18, 310-333. [CrossRef]

91. Nagarajan, K.; Saravanararaja, M.; Aruna Devi, P.S. Antibacterial Efficiency of Fabaceae Plants of a Tropical Freshwater Lake. ScieXplore Int. J. Res. Sci. 2015, 2, 13. [CrossRef]

92. Lee, Y.H.; Choo, C.; Watawana, M.I.; Jayawardena, N.; Waisundara, V.Y. An appraisal of eighteen commonly consumed edible plants as functional food based on their antioxidant and starch hydrolase inhibitory activities. J. Sci. Food Agric. 2015, 95, 2956-2964. [CrossRef] [PubMed]

93. Ittiyavirah, S.P.; Pullochal, I. Adaptogenic and nootropic activity of Mimosa pudica in albino wistar rats. Int. J. Nutr. Pharmacol. Neurol. Dis. 2014, 4, 231. [CrossRef]

94. Ao, S.; Kc, O.; Ukwueze, S. Evaluation of the antidiabetic potential of ethanol root extract of Mimosa pigra Linn (Fabaceae) in alloxan-induced diabetic albino rats. Int. J. Curr. Res. 2015, 7, 15577-15581.

95. Shorinwa Olusayo, A.; Nnamdi, E.; Afieroho Ozadheoghene, E. Evaluation of mimosa pigra roots on haematological and biochemical parameters of albino rats. World J. Pharm. Res. 2016, 5, 810-822.

96. Rosado-Vallado, M.; Brito-Loeza, W.; Mena-Rejon, G.; Quintero-Marmol, E.; Flores-Guido, J. Antimicrobial activity of Fabaceae species used in Yucatan traditional medicine. Fitoterapia 2000, 71, 570-573. [CrossRef] 
97. Saxena, R.; Sharma, R.; Nandy, B.; Jasuja, D.N. Qualitative and quantitative estimation of bioactive compounds in Mimosa hamata. Int. J. Pharm. Pharm. Sci. 2014, 6, 72-75.

98. Manosroi, J.; Zaruwa, M.; Manosroi, A. Potent Hypoglycemic Effect of Nigerian Anti-Diabetic Medicinal Plants. J. Complement. Integr. Med. 2011, 8, 1-14. [CrossRef] [PubMed]

99. Jiménez, N.; Carrillo-Hormaza, L.; Pujol, A.; Álzate, F.; Osorio, E.; Lara-Guzman, O. Antioxidant capacity and phenolic content of commonly used anti-inflammatory medicinal plants in Colombia. Ind. Crops Prod. 2015, 70, 272-279. [CrossRef]

100. Seraglio, S.K.T.; Valese, A.C.; Daguer, H.; Bergamo, G.; Azevedo, M.S.; Gonzaga, L.V.; Fett, R.; Costa, A.C.O. Development and validation of a LC-ESI-MS/MS method for the determination of phenolic compounds in honeydew honeys with the diluted-and-shoot approach. Food Res. Int. 2016, 87, 60-67. [CrossRef]

101. Nandipati, M.C.; Sumalatha, G.; Baburao, C.; Babu, J.R.; Sridevi, C. Antitumor activity of mimosa rubicaulis lam against ehrlich ascites carcinoma in swiss albino mice. Int. J. Pharm. Sci. Res. 2014, 5, 1514.

102. Wadood, A.; Ghufran, M.; Jamal, S.B.; Naeem, M.; Khan, A.; Ghaffar, R. Phytochemical analysis of medicinal plants occurring in local area of Mardan. Biochem. Anal Biochem. 2013, 2, 4. [CrossRef]

103. Dominguez, X.A.; Garcia, G.S.; Williams, H.J.; Ortiz, C.; Scott, A.I.; Reibenspies, J.H. Kukulkanins A and B, new chalcones from Mimosa tenuefolia. J. Nat. Prod. 1989, 52, 864-867. [CrossRef]

104. Bautista, E.; Calzada, F.; Ortega, A.; Yépez-Mulia, L. Antiprotozoal activity of flavonoids isolated from Mimosa tenuiflora (Fabaceae-Mimosoideae). J. Mex. Chem. Soc. 2011, 55, 251-253.

105. León, L.; Maldonado, E.; Cruz, A.; Ortega, A. Tenuiflorins A-C: New 2-Phenoxychromones from the Leaves of Mimosa tenuiflora. Planta Med. 2004, 70, 536-539. [CrossRef] [PubMed]

106. Gardner, D.; Riet-Correa, F.; Lemos, D.; Welch, K.; Pfister, J.; Panter, K. Teratogenic effects of Mimosa tenuiflora in a rat model and possible role of N-methyl-and N, N-dimethyltryptamine. J. Agric. Food Chem. 2014, 62, 7398-7401. [CrossRef]

107. Meckes-Lozoya, M.; Lozoya, X.; Marles, R.J.; Soucy-Breau, C.; Sen, A.; Arnason, J.T. N, N-dimethyltryptamine alkaloid in Mimosa tenuiflora bark (tepescohuite). Arch. Investig. Med. 1990, 21, 175.

108. Anton, R.; Jiang, Y.; Weniger, B.; Beck, J.; Rivier, L. Pharmacognosy of Mimosa tenuiflora (willd.) poiret. J. Ethnopharmacol. 1993, 38, 145-152. [CrossRef]

109. Jiang, Y.; Weniger, B.; Haag-Berrurier, M.; Anton, R.; Beck, J.P.; Italiano, L. Effects of saponins from Mimosa tenuiflora on lymphoma cells and lymphocytes. Phytother. Res. 1992, 6, 310-313. [CrossRef]

110. Vepsäläinen, J.J.; Auriola, S.; Tukiainen, M.; Ropponen, N.; Callaway, J. Isolation and characterization of yuremamine, a new phytoindole. Planta Med. 2005, 71, 1053-1057. [CrossRef] [PubMed]

111. Calvert, M.B.; Sperry, J. Bioinspired total synthesis and structural revision of yuremamine, an alkaloid from the entheogenic plant Mimosa tenuiflora. Chem. Commun. 2015, 51, 6202-6205. [CrossRef]

112. Okonkwo, C.J.; Njoku, O.U.; Okonkwo, T.J.; Afieroho, O.E.; Proksch, P. Two new acylated flavonol glycosides from Mimosa pigra L. leaves sub-family Mimosoideae. Future J. Pharm. Sci. 2016, 2, 71-75. [CrossRef]

113. Englert, J.; Weniger, B.; Lobstein, A.; Anton, R.; Krempp, E.; Guillaume, D.; Leroy, Y. Triterpenoid saponins from Mimosa pigra. J. Nat. Prod. 1995, 58, 1265-1269. [CrossRef]

114. Rakotomalala, G.; Agard, C.; Tonnerre, P.; Tesse, A.; Derbré, S.; Michalet, S.; Hamzaoui, J.; Rio, M.; Cario-Toumaniantz, C.; Richomme, P. Extract from Mimosa pigra attenuates chronic experimental pulmonary hypertension. J. Ethnopharmacol. 2013, 148, 106-116. [CrossRef] [PubMed]

115. Santos, M.; Moura, L.; Mendes, M.; Arcanjo, D.; Monção, N.; Araújo, B.; Lopes, J.; Silva-Filho, J.; Fernandes, R.; Oliveira, R. Hypotensive and vasorelaxant effects induced by the ethanolic extract of the Mimosa caesalpiniifolia Benth.(Mimosaceae) inflorescences in normotensive rats. J. Ethnopharmacol. 2015, 164, 120-128. [CrossRef] [PubMed]

116. Silva, M.; Carvalho, A.; Rocha, C.; Vilegas, W.; Silva, M.; Gouvêa, C. Ethanolic extract of Mimosa caesalpiniifolia leaves: Chemical characterization and cytotoxic effect on human breast cancer MCF-7 cell line. S. Afr. J. Bot. 2014, 93, 64-69. [CrossRef]

117. Jain, R.; Arora, R.; Jain, S. Saponins from the Roots of Mimosa hamata Willd. ChemInform 1997, 28, 61-64. [CrossRef]

118. Jain, R.; Arora, R.; Alam, S.; Jain, S. Pharmacological evaluation of Mimosa hamata roots. Fitoterapia 1997, 68, 377-378.

119. Jain, R.; Jain, S. Studies on Antimicrobial and Antioxidant Potentials of Triterpenoidal Saponins from Mimosa Hamata Willd. Int. J. Pharm. Phytopharm. Res. 2015, 4, 337-339.

120. Chiou, C.-T.; Shen, C.-C.; Tsai, T.-H.; Chen, Y.-J.; Lin, L.-C. Meroterpenoids and Chalcone-Lignoids from the Roots of Mimosa diplotricha. J. Nat. Prod. 2016, 79, 2439-2445. [CrossRef]

121. Camargo, L.M.D.M.; Férézou, J.-P.; Tinoco, L.W.; Kaiser, C.R.; Costa, S.S. Flavonoids from Mimosa xanthocentra (Leguminosae:

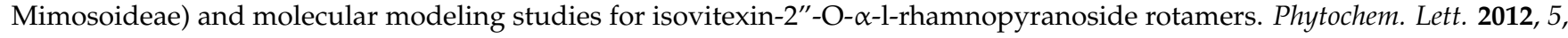
427-431. [CrossRef]

122. Pachter, I.J.; Zacharias, D.E.; Ribeiro, O. Indole alkaloids of Acer saccharinum (the Silver Maple), Dictyoloma incanescens, Piptadenia colubrina, and Mimosa hostilis. J. Org. Chem. 1959, 24, 1285-1287. [CrossRef]

123. Chrestani, F.; Sierakowski, M.R.; de Andrade Uchoa, D.E.; Nozawa, C.; Sassaki, G.L.; Gorin, P.A.J.; Ono, L. In vitro antiherpetic and antirotaviral activities of a sulfate prepared from Mimosa scabrella galactomannan. Int. J. Biol. Macromol. 2009, 45, $453-457$. [CrossRef]

124. Gupta, M.; Arias, T.; Etheart, J.; Hatfield, G. The occurrence of tryptamine and N-methyltryptamine in Mimosa somnians. J. Nat. Prod. 1979, 42, 234-236. [CrossRef] 
125. Thompson, J.F.; Morris, C.J.; Smith, I.K. New naturally occurring amino acids. Annu. Rev. Biochem. 1969, 38, 137-158. [CrossRef]

126. Chukwu, O.J.; Ahuchaogu, A.A.; Ukaogo, P.; Obike, A.; Echeme, J.B.O. Antifungal Activity of Mimosa pudica, Isolation and NMR Characterization of Bioactive Components. Asian J. Chem. Sci. 2017, 2, 1-5. [CrossRef]

127. Tsurumi, S.; Asahi, Y. Identification of jasmonic acid in Mimosa pudica and its inhibitory effect on auxin-and light-induced opening of the pulvinules. Physiol. Plant. 1985, 64, 207-211. [CrossRef]

128. Yuan, K.; Lü, J.; Yin, M. Chemical constituents of C-glycosylflavones from Mimosa pudica. Yao Xue Xue Bao Acta Pharm. Sin. 2006, 41, 435-438.

129. Yuan, K.; Lu, J.L.; Jia, A.; Zhu, J.X. Two new C-glycosylflavones from Mimosa pudica. Chin. Chem. Lett. 2007, 18, 1231-1234. [CrossRef]

130. Misra, A.; Tiwari, H. Constituents of roots of Boerhaavia diffusa. Phytochemistry 1971, 10, 3318-3319. [CrossRef]

131. Rajalakshmi, K.; Banu, N. Antimicrobial activity of natural chlorophyllin from endangered medicinal plant Mimosa pudica L. Int. J. Pharm. Pharm. Sci. 2016, 8, 387-389.

132. Josewin, B.; Ramachandrapai, M.; Suseelan, M. A new phenolic ketone from the leaves of Mimosa pudica Linn. Indian J. Chem. 1999, 38B, 251-253.

133. Kirk, L.F.; Møller, M.V.; Christensen, J.; Stærk, D.; Ekpe, P.; Jaroszewski, J.W. A 5-deoxyflavonol derivative in Mimosa pudica. Biochem. Syst. Ecol. 2003, 31, 103-106. [CrossRef]

134. Ueda, M.; Yamamura, S. Leaf-opening substance of Mimosa pudica L.; chemical studies on the other leaf movement of mimosa. Tetrahedron Lett. 1999, 40, 353-356. [CrossRef]

135. Ueda, M.; Yamamura, S. Leaf-closing substance of Mimosa pudica L.; chemical studies on another leaf-movement of mimosa II. Tetrahedron Lett. 1999, 40, 2981-2984. [CrossRef]

136. Ueda, M.; Yamamura, S. The chemistry of leaf-movement in Mimosa pudica L. Tetrahedron 1999, 55, 10937-10948. [CrossRef]

137. Pal, M.; Roychaudhury, A.; Pal, A.; Biswas, S. A novel tubulin from Mimosa pudica: Purification and characterization. Eur. J. Biochem. 1990, 192, 329-335. [CrossRef] [PubMed]

138. Khare, C. Encyclopedia of Indian Medicinal Plants: Rational Western Therapy, Ayurvedic and Other Traditional Usage, Botany; Springer: Berlin/Heidelberg, Germany, 2004.

139. Zhang, J.; Yuan, K.; Zhou, W.-1.; Zhou, J.; Yang, P. Studies on the active components and antioxidant activities of the extracts of Mimosa pudica Linn. from southern China. Pharmacogn. Mag. 2011, 7, 35.

140. Kang, J.G.; Shin, S.Y.; Kim, M.J.; Bajpai, V.; Maheshwari, D.K.; Kang, S.C. Isolation and Anti-fungal Activities of 2-Hydroxymethylchroman-4-one Produced by Burkholderia sp. MSSP. J. Antibiot. 2004, 57, 726-731. [CrossRef] [PubMed]

141. Dinda, B.; Ghosh, B.; Arima, S.; Sato, N.; Harigaya, Y. Steroids and terpenoid from Mimosa pudica roots. J. Indian Chem. Soc. 2006, 83, 1044-1046. [CrossRef]

142. Shu, W.-J.; Ho, J.-C. Two new antimicrobial diterpenoids from the roots of Mimosa pudica. J. Chin. Med. 2013, $24,223-229$.

143. Chatterjee, A.; Pakrashi, S.C. The Treatise on Indian Medicinal Plants; Publication and Information Directorate: New Delhi, India, 1991; Volume 3.

144. Yadava, R.; Yadav, S. A novel bufadienolide from the seeds of Mimosa pudica Linn. Asian J. Chem. 2001, 13, 1157-1160.

145. Zaware, B.; Chaudhari, S.; Shinde, M. An overview of Mimosa pudica Linn. Chem. Pharmacol. Profile. Res. J. Pharm. Biol. Chem. Sci. 2014, 5, 754-761.

146. Yeung, A.W.K.; Tzvetkov, N.T.; Georgieva, M.G.; Ognyanov, I.V.; Kordos, K.; Jóźwik, A.; Kühl, T.; Perry, G.; Petralia, M.C.; Mazzon, E.; et al. Reactive Oxygen Species and Their Impact in Neurodegenerative Diseases: Literature Landscape Analysis. Antioxid. Redox Signal. 2020, 34, 402-420. [CrossRef]

147. Sueishi, Y.; Nii, R. A comparative study of the antioxidant profiles of olive fruit and leaf extracts against five reactive oxygen species as measured with a multiple free-radical scavenging method. J. Food Sci. 2020, 85, 2737-2744. [CrossRef] [PubMed]

148. Gupta, R.K.; Rathi, R.B. Evaluation of Anticancer Action of Martynia annua Linn Root Extract on Different Human Cancer Cell Lines. J. Pharm. Res. Int. 2021, 33, 96-109. [CrossRef]

149. Khan, T.; Ali, M.; Khan, A.; Nisar, P.; Jan, S.A.; Afridi, S.; Shinwari, Z.K. Anticancer plants: A review of the active phytochemicals, applications in animal models, and regulatory aspects. Biomolecules 2020, 10, 47. [CrossRef] [PubMed] 